JOURNAL OF

SYMPLECTIC GEOMETRY

Volume 6, Number 2, 159-187, 2008

\title{
LENGTH MINIMIZING PATHS IN THE HAMILTONIAN DIFFEOMORPHISM GROUP
}

\author{
Peter W. Spaeth
}

\begin{abstract}
On any closed symplectic manifold, we construct a path-connected neighborhood of the identity in the Hamiltonian diffeomorphism group with the property that each Hamiltonian diffeomorphism in this neighborhood admits a Hofer and spectral length minimizing path to the identity. This neighborhood is open in the $\boldsymbol{C}^{1}$-topology. The construction utilizes a continuation argument and chain level result in the Floer theory of Lagrangian intersections.
\end{abstract}

\section{Introduction}

First, we prove a chain level result in the Floer theory of Lagrangian intersections, Theorem A, that relates the local Floer cap product to the so-called thin part of the Floer cap product. The proof relies upon Chekanov's version [2] of the Floer continuation argument and a generalized definition of chain homotopy, called a $\lambda$-homotopy. Together with an algebraic result, Proposition 2.3, this provides the existence of a certain pseudo-holomorphic curve with prescribed asymptotics, see Proposition 4.2. The existence of this curve yields numerical identities among certain non-degenerate Hamiltonian diffeomorphisms, see Theorem B. Consequently, we achieve the main goal of the article, namely that the Hamiltonian group of any closed symplectic manifold exhibits a local flatness property under the Hofer and spectral norms, see Corollary C and Corollary D. Under the Hofer norm, Bialy and Polterovich [1] observe this for a neighborhood of the identity of $C^{1}$-small Hamiltonians in the group of compactly supported Hamiltonian diffeomorphisms of $\mathbb{R}^{2 n}$. Lalonde and McDuff [10] and McDuff [12] also obtain the $C^{1}$-small flatness on an arbitrary symplectic manifold $(M, \omega)$ under the Hofer norm. Recently, Oh $[\mathbf{2 1}]$ proves the $C^{1}$-small flatness for the Hofer and spectral norms.

The neighborhood of the identity we construct is likely larger than has appeared in the literature. It consists of all engulfable Hamiltonians for 
which the oscillation of the generating function is smaller than half the minimal energy of a non-constant pseudo-holomorphic sphere in $M$. This is an open set in the $C^{1}$-topology. Engulfability is defined in Definition 1.3.

1.1. Statement of Theorem A. Let $(P, \omega)$ be any tame symplectic manifold (not necessarily closed). This means that there is an $\omega$-tame almost complex structure $J_{0}$ on $P$ such that the corresponding metric $g_{J_{0}}$ has bounded curvature and whose injectivity radius is bounded from zero from below. Examples include all closed symplectic manifolds, the cotangent bundle of any closed manifold with the canonical symplectic structure and $\mathbb{R}^{2 n}$ with its standard symplectic structure.

Let $L$ be a connected, closed Lagrangian submanifold of $P$ and fix a Darboux-Weinstein neighborhood $\mathcal{U}$ of $L$. Denote by $\sigma(L ; P, \omega)$ the minimal area of any non-constant pseudo-holomorphic sphere or disk with boundary on $L$ in $P$. Let $H: P \times[0,1] \rightarrow \mathbb{R}$ be a smooth Hamiltonian function. Assume that $H$ is normalized, which means that for some compact set $K \subset P$,

$$
\text { supp } H(\cdot, t) \subset K, \quad \text { for all } t \in[0,1] .
$$

In addition, assume that $H$ is non-degenerate; that is

$$
\phi_{H}^{1}(L) \pitchfork L .
$$

Here $\phi_{H}^{1}$ denotes the time-one map of Hamilton's equation

$$
\dot{z}=X_{H}(z, t), \quad z(0), z(1) \in L
$$

where the Hamiltonian vector field $X_{H}$ is defined by the equation

$$
\left.X_{H}\right\lrcorner \omega=d H .
$$

Now suppose $s \in \mathbb{R}$ satisfies $0<s \ll 1$ and we now consider the Hamiltonian function $s \cdot H: P \times[0,1] \rightarrow \mathbb{R}$. We recall the set-up to the Floer theory $H F\left(L, s H ; \mathbb{Z}_{2}\right)$ beginning with the action functional. Fix a reference path $l_{0}$ on $L$. The action functional $\mathcal{A}_{s H}$ is defined on the relative analog of Hofer and Salamon's [8] Novikov covering $\widetilde{\Omega}_{0}\left(L, l_{0} ; P, \omega\right)=\widetilde{\Omega}_{0} \stackrel{\pi}{\rightarrow} \Omega_{0}$ over the space $\Omega_{0}$ of smooth contractible paths beginning and ending on $L$

$$
\Omega_{0}=\left\{l:[0,1] \longrightarrow P \mid l(0), l(1) \in L, l \text { homotopic rel } L \text { to } l_{0}\right\} .
$$

$\widetilde{\Omega}_{0}$ consists of equivalence classes $[l, \tilde{l}]$ where the map $\tilde{l}:[0,1] \times[0,1] \rightarrow P$ provides the homotopy from $l_{0}$ to $l$

$$
\tilde{l}(0, t)=l_{0}(t), \quad \tilde{l}(1, t)=l(t), \quad \tilde{l}(s, 0) \in L \quad \text { and } \quad \tilde{l}(s, 1) \in L .
$$

Two pairs $\left(l_{1}, \widetilde{l_{1}}\right)$ and $\left(l_{2}, \widetilde{l_{2}}\right)$ are by definition equivalent if both $l_{1}=l_{2}$ and the connected sum along $l, \widetilde{l_{1}} \# \widetilde{\widetilde{l_{2}}}$, with the reversed orientation on $\widetilde{l_{2}}$ satisfies

$$
\mu_{L}\left(\widetilde{l_{1}} \# \overline{\widetilde{l_{2}}}\right)=0=I_{\omega}\left(\widetilde{l_{1}} \# \overline{\widetilde{l_{2}}}\right)
$$


where $\mu_{L}$ denotes the Maslov index and $I_{\omega}$ the symplectic action homomorphism on the relative homotopy group $\pi_{2}(P, L)$. The quotient group

$$
\Gamma=\frac{\pi_{2}(P, L)}{\operatorname{ker} \mu_{L} \cap \operatorname{ker} I_{\omega}}
$$

acts on elements $[l, \tilde{l}] \in \widetilde{\Omega}$ by gluing a disk along the reference path $l_{0}$. Moreover $\Gamma$ is a free abelian group.

The action functional is defined as follows

$$
\mathcal{A}_{s H}([l, \tilde{l}])=-\int_{[0,1]^{2}} \tilde{l}^{*} \omega-\int_{0}^{1} H(l(t), t) d t .
$$

Critical points $[z, \tilde{z}]$ of the action functional consist of pairs

$$
\operatorname{Crit}\left(\mathcal{A}_{s H}\right)=\left\{[z, \tilde{z}] \in \widetilde{\Omega}_{0} \mid z \text { satisfies }(1.1)\right\} .
$$

Typically we will denote elements of the covering space $[l, \tilde{l}]$ or $[l, w]$ while critical points will be denoted $[z, \tilde{z}]$ or $[z, w]$. Denote $C F_{*}(L, s H)$ the vector space of Floer chains generated over $\mathbb{Z}_{2}$. The solutions to equation (1.1) are in one to one correspondence with the points of intersection of $L$ and $\phi_{s H}^{1}(L)$.

Fix a time-dependent almost complex structure $J=J_{t}$ on $P$. The relative Floer differential is defined by counting finite energy solutions $u: \mathbb{R} \times[0,1] \rightarrow$ $P$ to the equation

$$
\left\{\begin{array}{l}
\frac{\partial u}{\partial \tau}+J(u, t)\left(\frac{\partial u}{\partial t}-X_{s H}(u, t)\right)=0 \\
u(\tau, 0), u(\tau, 1) \in L
\end{array}\right.
$$

where the energy is defined to be

$$
E_{J}(u):=\int_{-\infty}^{+\infty}\left|\frac{\partial u}{\partial \tau}\right|^{2} d \tau=\int_{-\infty}^{+\infty} \int_{0}^{1} \omega\left(\frac{\partial u}{\partial \tau}, J \frac{\partial u}{\partial \tau}\right) d t d \tau
$$

with respect to the metric $g_{J}=\omega(\cdot, J \cdot)$. The energy of a solution $u$ to equation (1.2) can also be written

$$
\frac{1}{2} \int_{-\infty}^{+\infty}\left|\frac{\partial u}{\partial \tau}\right|^{2}+\left|\frac{\partial u}{\partial t}-X_{H}(u, t)\right|^{2} d \tau
$$

For generic $s H$, the finite energy solutions of equation (1.2) converge exponentially to solutions of equation (1.1) (see [24]). We denote the collection of finite energy solutions

$$
\mathcal{N}(L, s H ; J)=\left\{u: \mathbb{R} \times[0,1] \rightarrow P \mid u \text { satisfies }(1.2) \text { and } E_{J}(u)<\infty\right\}
$$

and when asymptotic conditions are specified

$$
\lim _{\tau \rightarrow-\infty} u(\tau, \cdot)=[x, \tilde{x}], \quad \lim _{\tau \rightarrow+\infty} u(\tau, \cdot)=[y, \tilde{y}] \text { with } \tilde{x} \# u \simeq \widetilde{y},
$$

we write $\mathcal{N}([x, \tilde{x}],[y, \tilde{y}] ; J)$. 
For a general Lagrangian submanifold $L$, the relative Floer differential may be obstructed or not square to zero; however, when $s$ is sufficiently small and $J$ is sufficiently close to an autonomous almost complex structure, the solutions to equation (1.2) are either thin (written $u \in \mathcal{N}^{\circ}$ ) or thick (resp. $u \in \mathcal{N}^{\prime}$ ) (see also Proposition 3.1 below, Proposition 4.1 [13] and Lemma 6 $[2])$. The thin part of the Floer differential, $\partial^{\circ}$, is defined by counting the thin solutions to equation (1.2). And in fact (again when $s$ is sufficiently small and $J$ is close to an autonomous almost complex structure)

$$
\partial^{\circ} \circ \partial^{\circ}=0, \quad H_{*}\left(\partial^{\circ}\right)=H F_{*}^{\circ}\left(L, s H ; \mathbb{Z}_{2}\right) \cong H_{*}\left(L ; \mathbb{Z}_{2}\right),
$$

see [2, Lemma 7] and [13, Theorem 4.7].

The Floer cap product is defined by counting solutions to equation (1.2) passing through a marked point $p \in L$. The collection of these maps we denote

$$
\mathcal{N}(L, s H ; J ; p)=\{u \in \mathcal{N}(L, s H ; J) \mid u(0,0)=p \in L\} .
$$

Again there is a thick and thin decomposition and the thin Floer cap product is defined by counting thin elements $u \in \mathcal{N}^{\circ}(L, s H ; J ; p)$. The thin part of the Floer cap product, for generic $p \in L$, descends to an isomorphism on $H F_{*}^{\circ}\left(L, s H ; \mathbb{Z}_{2}\right)$, see $[\mathbf{1 5}$, Lemma 7.4$]$.

Next we could also define a local Floer cap product by counting those curves $u \in \mathcal{N}(L, s H ; J ; p)$ as above whose image lies in the Darboux neighborhood $\mathcal{U}$ of $L$. These local curves we denote $\mathcal{N}^{\mathcal{U}}(L, s H ; J, p)$. While we do not know if this local Floer cap product is an isomorphism, it is related to the thin cap action.

Indeed let

$$
\begin{aligned}
\Phi^{+}: C F_{*}\left(L, s H ; \mathbb{Z}_{2}\right) & \longrightarrow C F_{*}\left(L, 1 \cdot H ; \mathbb{Z}_{2}\right) \\
\Phi^{-}: C F_{*}\left(L, 1 \cdot H ; \mathbb{Z}_{2}\right) & \longrightarrow C F_{*}\left(L, s H ; \mathbb{Z}_{2}\right)
\end{aligned}
$$

be the usual continuation maps in the Floer theory defined by the Floer continuation equation (see Section 3). Recall that in [2], Chekanov proves that if the Hofer length, $\|H\|$, of $\mathrm{H}$ is less than $\sigma(L ; P, \omega)$, then for $s$ sufficiently small and generic $H$, the identity map and the composition $\Phi^{-} \circ \Phi^{+}$

$$
\mathrm{id}, \Phi^{-} \circ \Phi^{+}: C F_{*}\left(L, s H ; \mathbb{Z}_{2}\right) \longrightarrow C F_{*}\left(L, s H ; \mathbb{Z}_{2}\right)
$$

are $\lambda$-homotopic, see Section 2 for a review of $\lambda$-homotopy.

This motivated us to prove that the maps $[L] \cap_{s}^{\circ}(\cdot)$ and $\Phi^{-} \circ[L] \cap^{\mathcal{U}}(\cdot) \circ \Phi^{+}$

$$
[L] \cap_{s}^{\circ}(\cdot), \Phi^{-} \circ\left([L] \cap^{\mathcal{U}}(\cdot)\right) \circ \Phi^{+}: C F_{s}\left(L, s H ; \mathbb{Z}_{2}\right) \rightarrow C F_{s}\left(L, s H ; \mathbb{Z}_{2}\right)
$$

are $\lambda$-homotopic under a slightly stronger assumption on $\|H\|$.

Theorem A. Suppose that the Hamiltonian diffeomorphism $\phi=\phi_{H}^{1}$ is non-degenerate in the sense that $\phi_{H}^{1}(L)$ meets $L$ transversally, $\phi_{H}^{1}(L) \pitchfork L$, 
and satisfies

$$
\|H\|<\frac{\sigma(L ; P, \omega)}{2}
$$

where $\|H\|$ is Hofer's length of $H$. For s sufficiently small, the thin Floer cap product

$$
[L] \cap_{s}^{\circ}(\cdot): C F_{*}(L, s H) \longrightarrow C F_{*}(L, s H)
$$

is $\lambda$-homotopic to the composition

$$
\Phi^{-} \circ[L] \cap^{\mathcal{U}}(\cdot) \circ \Phi^{+}: C F_{*}(L, s H) \longrightarrow C F_{*}(L, s H) .
$$

The proof of Theorem A is the subject of Section 3 .

1.2. Theorem $\mathbf{B}$ and its consequences. Let $(M, \omega)$ be a closed symplectic manifold of dimension $2 n$ and $H: M \times[0,1] \rightarrow \mathbb{R}$ be any smooth Hamiltonian function. The Hamiltonian vector field $X_{H}$ generates a Hamiltonian flow $\phi_{H}^{t}$; that is,

$$
\frac{d}{d t} \phi_{H}^{t}=X_{H} \circ \phi_{H}^{t}, \quad \phi_{H}^{0}=\mathrm{id} .
$$

We abbreviate this by writing $H \mapsto \phi_{H}^{t}$ or $H \mapsto \phi$ if we are interested in the time-one map. Also we denote the isotopy $t \in[0,1] \mapsto \phi_{H}^{t}$ by $\phi_{H}$.

Given a second Hamiltonian function $G: M \times[0,1] \rightarrow \mathbb{R}$ with $G \mapsto \psi_{G}^{t}$, the chain rule

implies that the Hamiltonian function

$$
\frac{d}{d t}\left(\phi_{H}^{t} \circ \psi_{G}^{t}\right)=\left(\dot{\phi_{H}^{t}}\right)+T \phi_{H}^{t}\left(\dot{\psi}_{H}^{t}\right)
$$

$$
H \# G(x, t):=H(x, t)+G\left(\left(\phi_{H}^{t}\right)^{-1}(x), t\right)
$$

generates the composed isotopy $t \mapsto \phi_{H}^{t} \circ \psi_{G}^{t}$ and that the Hamiltonian function

$$
\bar{H}(x, t):=-H\left(\phi_{H}^{t} x, t\right)
$$

generates the inverse flow $t \mapsto\left(\phi_{H}^{t}\right)^{-1}$. In other words

$$
\left\{\begin{array}{l}
H \# G:=H+G \circ\left(\phi_{H}^{t}\right)^{-1} \mapsto \phi_{H}^{t} \circ \psi_{H}^{t} \\
\bar{H}=-H \circ \phi_{H}^{t} \mapsto\left(\phi_{H}^{t}\right)^{-1} .
\end{array}\right.
$$

When the symplectic manifold is closed, a Hamiltonian function $H: M \times$ $[0,1] \rightarrow \mathbb{R}$ is normalized if its mean value is zero for each time $t$

$$
\int_{M} H(x, t) d \mu(x)=0 \quad \text { for all } t \in[0,1],
$$

where $d \mu=\omega^{n} / n$ !. The Hofer length of a Hamiltonian isotopy $\phi_{H}$, which does not depend on the normalization, is the mean value (over time) of the oscillation of the Hamiltonian function $H$

$$
\operatorname{length}\left(\phi_{H}^{t}\right)=\|H\|=\int_{0}^{1} \operatorname{osc}_{x \in M} H(x, t) d t,
$$


where for each $t \in[0,1]$

$$
\operatorname{osc}_{x \in M}(H(x, t))=\max _{x \in M} H(x, t)-\min _{x \in M} H(x, t) .
$$

Then the Hofer norm of the Hamiltonian diffeomorphism $\phi$ is defined to be

$$
\|\phi\|_{\text {Hofer }}=\inf _{H \mapsto \phi}\|H\| \text {. }
$$

Theorem 1.1 (Hofer [7], Polterovich [23], Lalonde and McDuff [9]). The function $\|\cdot\|_{\text {Hofer }}: \operatorname{Ham}(M, \omega) \rightarrow \mathbb{R}_{+}$satisfies the following properties. Let $\phi, \psi \in \operatorname{Ham}(M, \omega)$.

1) $\left\|\theta \phi \theta^{-1}\right\|=\|\phi\|$ for any $\theta \in \operatorname{Symp}(M, \omega)$ (symplectic invariance).

2) $\|\phi\|=\left\|\phi^{-1}\right\|$ (symmetry).

3) $\|\phi \psi\| \leq\|\phi\|+\|\psi\|$ (triangle inequality).

4) $\|\phi\|=0$ if and only if $\phi$ is the identity (non-degeneracy).

Hofer [7] proves that $d(\phi, \psi)=\left\|\phi \circ \psi^{-1}\right\|_{\text {Hofer }}$ defines a bi-invariant nondegenerate distance on the group of compactly supported Hamiltonian diffeomorphisms of $\mathbb{R}^{2 n}$ with its standard symplectic structure using infinite dimensional variational methods. Viterbo [29] later proves this result with generating functions. Polterovich [23] further generalizes this result to all tame, rational symplectic manifolds using pseudo-holomorphic curves and finally Lalonde and McDuff [9] prove the non-degeneracy property, also via pseudo-holomorphic curves, for any symplectic manifold. Chekanov [2] and Oh [14] prove the non-degeneracy property for tame symplectic manifolds via Floer-theoretical techniques.

The association from a Hamiltonian flow to normalized Hamiltonian function is injective. The normalization is important when studying the critical values of the associated symplectic action functional. For instance the action spectrum, $\operatorname{Spec}(H)$, i.e., the set of critical values of the action functional $\mathcal{A}_{H}$ depends only on the homotopy class $[\phi, H]$, see $[\mathbf{2 0}]$. And in fact if $(M, \omega)$ is symplectically aspherical, Schwarz $[\mathbf{2 6}]$ proves $\operatorname{Spec}(H)$ depends only on the time-one map $\phi$.

Now in order to review the spectral invariants (see [16] and [21] for a precise treatment) assume temporarily that the Hamiltonian function $H$ is 1-periodic in time, $H: M \times \mathbb{R} / \mathbb{Z} \rightarrow \mathbb{R}$. Let $\Omega_{0}(M)$ denote the space of smooth contractible loops $l: S^{1} \rightarrow M$ and $\widetilde{\Omega}_{0}(M) \rightarrow \Omega_{0}(M)$ denote Hofer and Salamon's [8] covering space on which the symplectic action functional is defined. In the closed string setting, $\widetilde{\Omega}_{0}(M)$ consists of equivalence classes of pairs $(l, w)$, where $w$ is a disk bounding $l$. Let $w \# \overline{w^{\prime}}$ denote the sphere obtained from gluing two disks $w$ and $w^{\prime}$ (with the opposite orientation) along a common boundary. Two pairs $(l, w)$ and $\left(l^{\prime}, w^{\prime}\right)$ are said to be $\Gamma$-equivalent if and only if

$$
l=l^{\prime} \quad \text { and } \quad \int_{S^{2}}\left(w \# \overline{w^{\prime}}\right)^{*} \omega=0=\int_{S^{2}}\left(w \# \overline{w^{\prime}}\right)^{*} c_{1}(\omega) .
$$


The automorphism group of the covering $\widetilde{\Omega}_{0}(M) \rightarrow \Omega_{0}(M)$

$$
\Gamma=\frac{\pi_{2}(M)}{\operatorname{ker} \omega \cap \operatorname{ker} c_{1}(\omega)}
$$

is called the Novikov covering group.

We define the action functional $\mathcal{A}_{H}: \widetilde{\Omega}_{0}(M) \rightarrow \mathbb{R}$ by setting

$$
\mathcal{A}_{H}([l, w])=-\int_{D^{2}} w^{*} \omega-\int_{0}^{1} H(l(t), t)=d t .
$$

The assignment $x \in M \mapsto \phi_{H}^{t}(x)$ is a one to one correspondence between the set of fixed points, $\operatorname{Fix}(\phi)$, of $\phi$ and $\operatorname{Per}\left(X_{H}\right)$, the set of 1-periodic orbits of the Hamiltonian vector field, $X_{H}$. A well-known calculation shows that the set of cricital points $\operatorname{Crit}\left(\mathcal{A}_{H}\right)$ is given by

$$
\operatorname{Crit}\left(\mathcal{A}_{H}\right)=\left\{[z, \tilde{z}] \in \widetilde{\Omega}_{0}(M) \mid z \in \operatorname{Per}\left(X_{H}\right)\right\} .
$$

Any constant loop $x \in \operatorname{Per}\left(X_{H}\right)$ admits the canonical constant bounding disk $\widehat{x}$.

The set of Floer-Novikov chains $C F_{*}(H)$ consists of all formal sums

$$
\alpha_{H}=\sum_{[z, \tilde{z}] \in \operatorname{Crit} \mathcal{A}_{H}} a_{[z, \tilde{z}]} \cdot[z, \tilde{z}], \quad a_{[z, \tilde{z}]} \in \mathbb{Q}
$$

such that

$$
\#\left\{[z, \tilde{z}] \mid a_{[z, \tilde{z}]} \neq 0 \text { and } \mathcal{A}_{H}([z, \tilde{z}]) \geq r\right\}<\infty
$$

for all $r \in \mathbb{R}$. If the critical point $[z, \tilde{z}]$ in equation (1.5) has a non-zero coefficient $a_{[z, \tilde{z}]}$, then we say that $[z, \tilde{z}]$ contributes to the chain $\alpha$ or write $[z, \tilde{z}] \in \alpha$.

Assume that $H$ is non-degenerate in the Floer-theoretical sense and denote the Conley-Zehnder index of a critical point $[z, \tilde{z}]$ by $\mu_{H}([z, \tilde{z}])$. The set of Floer-Novikov chains of index $n$ is denoted $C F_{n}(H)$.

The reader is referred to $[\mathbf{2 1}]$ for a more detailed account of the Floer homology theory of the action functional. Let $J_{0}$ denote an almost complex structure on $M$ and $J=J(\cdot, t)$ be a 1-periodic family of almost complex structures with $J(\cdot, 0)=J_{0}$. We only mention that the Floer differential is defined by counting finite energy solutions to the partial differential equation

$$
\left\{\begin{array}{l}
u:(\tau, t) \in \mathbb{R} \times S^{1} \rightarrow u(\tau, t) \in M \\
\frac{\partial u}{\partial \tau}+J(u, t)\left(\frac{\partial u}{\partial t}-X_{H}(u, t)\right)=0
\end{array}\right.
$$

Any non-zero quantum cohomology class $a \in Q H^{*}(M)$ determines a spectral invariant $\rho(H ; a) \in \mathbb{R}$ by the following mini-max procedure. Fix a Floer-Novikov cycle $\alpha$ Poincaré dual, written $[\alpha]^{b}=a$ (in a precise sense, see the "canonical Floer cycle," [19]) to $a$. Let its level, $\lambda_{H}(\alpha)$, be the maximum action of any critical point contributing to the cycle. Then $\rho(H ; a)$ is defined to be the infimal level among all cycles $\alpha$ dual to $a$. Great 
care must be made to show this number is finite, does not depend on the various choices involved (such as the almost complex structure) and satisfies a short list of axioms, see $[\mathbf{1 9}$, Theorem I].

The non-degenerate spectrality axiom (a theorem by the work of Oh $[\mathbf{1 7}]$ and Usher [27]) asserts that when $H$ is non-degenerate, $\rho(H ; a)$ is a critical value of the action functional $\mathcal{A}_{H}$. The non-degenerate spectrality axiom implies that $\rho(H ; a)$ only depends on the homotopy class of $\phi_{H}$ with fixed endpoints, which in turn allows the spectral invariant to be extended to any, not necessarily periodic, Hamiltonian function $H: M \times[0,1] \rightarrow \mathbb{R}$.

Furthermore, $\rho(H ; a)$ depends continuously on the Hamiltonian $H$ and so extends to any smooth function $H$, non-degenerate or not. The triangle inequality asserts that

$$
\rho(H \# K ; a \cdot b) \leq \rho(H ; a)+\rho(K ; b)
$$

for any two Hamiltonian functions $H$ and $K$ and where $a \cdot b$ refers to the quantum product of $a$ and $b$. Having said all of this, the normalization axiom then implies

$$
0=\rho(H \# \bar{H} ; 1) \leq \rho(H ; 1)+\rho(\bar{H} ; 1) .
$$

The former denotes the spectral length of the isotopy $\phi_{H}$,

$$
\operatorname{length}_{\gamma}\left(\phi_{H}\right)=\gamma(H)=\rho(H ; 1)+\rho(\bar{H} ; 1) .
$$

The time-reversal $t \mapsto 1-t$ provides another useful Hamiltonian function $\widetilde{H}(x, t):=-H(x, 1-t)$. The time-one mapping of the vector field $X_{\widetilde{H}}$ is also $\left(\phi_{H}^{1}\right)^{-1}$ and the two isotopies $\phi_{\bar{H}}^{t}$ and $\phi_{\widetilde{H}}^{t}$ are homotopic rel endpoints. Thus by the homotopy axiom $\rho(\bar{H} ; 1)=\rho(\widetilde{H} ; 1)[\mathbf{2 1}$, Lemma 5.2] and so the spectral length may be written as

$$
\gamma(H)=\rho(H ; 1)+\rho(\widetilde{H} ; 1) .
$$

The infimum over all isotopies ending at a given Hamiltonian $\phi$ defines the spectral norm

$$
\gamma(\phi):=\inf _{H \mapsto \phi} \gamma(H) .
$$

For the reader's convenience, we recall

Theorem 1.2 (Schwarz [26], Oh [21]). The spectral norm $\gamma: \operatorname{Ham}(M, \omega)$ $\rightarrow \mathbb{R}_{+}$satisfies the following properties. Let $\phi, \psi \in \operatorname{Ham}(M, \omega)$.

(1) $\gamma\left(\theta \phi \theta^{-1}\right)=\gamma(\phi)$ for any $\theta \in \operatorname{Symp}(M, \omega)$ (symplectic invariance).

(2) $\gamma(\phi)=\gamma\left(\phi^{-1}\right)$ (symmetry).

(3) $\gamma(\phi \psi) \leq \gamma(\phi)+\gamma(\psi)$ (triangle inequality).

(4) $\gamma(\phi)=0$ if and only if $\phi$ is the identity (non-degeneracy).

(5) $\gamma(\phi) \leq\|\phi\|_{\text {Hofer }}$ 
The spectral norm $\gamma$ defines a bi-invariant metric on the Hamiltonian group which is a Floer-theoretical refinement of the Hofer metric. In particular, the spectral norm is a lower bound for the Hofer norm.

Roughly speaking, the spectral norm of a Hamiltonian diffeomorphism $\phi_{H}$ is the smallest action difference among homologically essential critical values of the action functionals $\mathcal{A}_{H}$ and $\mathcal{A}_{\widetilde{H}}$ corresponding to critical points $[z, w]$ and $[\widetilde{z}, \widetilde{w}]$ with Conley-Zehnder indices $\mu_{H}([z, w])=n$ and $\mu_{\widetilde{H}}([\widetilde{z}, \widetilde{w}])=n$. For the moment we change the notation briefly and denote critical values of the action functionals $\mathcal{A}_{H}$ and $\mathcal{A}_{\widetilde{H}}$ by $[z, w]$ and $[\widetilde{z}, \widetilde{w}]$. Under Poincaré duality, such Floer cycles correspond with the quantum cohomology class 1.

To reflect the fact that the spectral metric involves only this cohomology class, Oh introduces the homological area $A(\phi ; 1)$ which is, again roughly speaking, the minimal energy of a Floer trajectory connecting the maximum and minimum of $H$. The time-reversal applied to the critical point $[\widetilde{z}, \widetilde{w}] \in$ Crit $\mathcal{A}_{\widetilde{H}}$ yields a critical point, say $\left[z^{\prime}, w^{\prime}\right] \in \operatorname{Crit} \mathcal{A}_{H}$ and $\mu_{H}\left[z^{\prime}, w^{\prime}\right]=-n$. Fix a point $q \in M$ and Floer cycles $\alpha_{H}$ and $\beta_{\widetilde{H}}$ of Conley-Zehnder indices $\mu_{H}([z, w])=n, \mu_{\widetilde{H}}([\widetilde{z}, \widetilde{w}])=n$.

Now consider the one-marked stable Floer trajectories $u$ satisfying the following data

$$
u=u_{1} \# \cdots \# u_{N}
$$

with each $u_{j}$ a Floer trajectory with finitely many sphere bubbles attached satisfying

$$
\left\{\begin{array}{l}
\frac{\partial u}{\partial \tau}+J(u, t)\left(\frac{\partial u}{\partial t}-X_{H}(u, t)\right)=0 \\
u_{1}(-\infty)=[z, w] \in \alpha_{H}, u_{N}(+\infty) \in\left[z^{\prime}, w^{\prime}\right] \text { with }[\widetilde{z}, \widetilde{w}] \in \beta_{\widetilde{H}} \\
u_{j}(0,0)=q \text { for some } j=1,2, \ldots, N
\end{array}\right.
$$

The energy $E_{J}(u)$ of a curve $u: \mathbb{R} \times S^{1} \rightarrow M$ satisfying equation (1.7) is

$$
\int_{-\infty}^{\infty}\left|\frac{\partial u}{\partial \tau}\right|^{2} d \tau
$$

with respect to the metric on $g_{J}=\omega(\cdot, J \cdot)$. The energy of such a $u=$ $u_{1} \# \cdots \# u_{N}$ is the sum

$$
\sum_{k} E_{J}\left(u_{k}\right)
$$

plus the symplectic area of any attached sphere bubbles. Now, the homological area is defined by the following mini-max procedure, reminiscent of the definition of $\sigma(M, \omega)$.

Begin by defining

$$
A\left(\phi, J_{0} ; J ; \alpha_{H}, \beta_{\widetilde{H}} ; q\right)=\inf \left\{E_{J}(u) \mid u \text { satisfies }(1.7)\right\}
$$

and then set

$$
A\left(\phi, J_{0} ; J ; 1 ; q\right)=\inf \left\{A\left(\phi, J_{0} ; J ; \alpha_{H}, \beta_{\widetilde{H}} ; q\right) \mid\left[\alpha_{H}\right]^{b}=1=\left[\beta_{\widetilde{H}}\right]^{b}\right\} .
$$


Now define

$$
\begin{aligned}
A\left(\phi, J_{0} ; J ; 1\right) & =\sup \left\{A\left(\phi, J_{0} ; J ; 1 ; q\right) \mid q \in M\right\} \\
A\left(\phi, J_{0} ; 1\right) & =\sup \left\{A\left(\phi, J_{0} ; J ; 1\right) \mid J\right\}
\end{aligned}
$$

and

$$
A(\phi ; 1)=\sup \left\{A\left(\phi, J_{0} ; 1\right) \mid J_{0}\right\} .
$$

Oh proves in Theorem $\mathrm{C}[\mathbf{2 1}]$ that any non-degenerate Hamiltonian diffeomorphism $\phi$, not necessarily engulfable, satisfies

$$
A(\phi ; 1) \leq \gamma(\phi) \leq\|\phi\|_{\text {Hofer }} .
$$

The graph $\Delta_{\phi}$ of a Hamiltonian diffeomorphism $\phi$ is a Lagrangian submanifold of the product $(M \times M,-\omega \oplus \omega)$. Let $\mathcal{U}$ be a Darboux neighborhood of the diagonal and $\Phi$ be a Darboux chart

$$
\Phi: \mathcal{U} \subset(M \times M,-\omega \oplus \omega) \rightarrow \mathcal{V}=\Phi(\mathcal{U}) \subset\left(T^{*}(\Delta), d \Lambda\right),
$$

where $\Phi^{*}(d \Lambda)=-\omega \oplus \omega$ and $\Lambda$ is the canonical one-form on the cotangent bundle $T^{*} \Delta$.

Recall that a Hamiltonian function $H$ is called quasi-autonomous [1] if there exist fixed points $x^{+}, x^{-} \in M$ with

$$
H\left(x^{+}, t\right)=\max _{x \in M} H(x, t) \quad \text { and } \quad H\left(x^{-}, t\right)=\min _{x \in M} H(x, t) .
$$

If $\phi_{F}$ is a Hofer geodesic, then necessarily $F$ must be quasi-autonomous, see $[\mathbf{1}, \mathbf{1 0}, \mathbf{2 8}]$.

Motivated by Laudenbach's work on Lagrangian submanifolds [11], Oh [21] introduces the following.

Definition and Lemma 1.3 (Oh [21]). A Hamiltonian diffeomorphism $\phi$ is engulfable if it is $C^{0}$-small in the sense that its graph is contained in a Darboux-Weinstein neighborhood of the diagonal and its image under the Darboux chart $\Phi$ within the cotangent bundle is the graph of an exact one-form, $d S_{\phi}$,

$$
\Phi\left(\Delta_{\phi}\right)=\operatorname{graph} d S_{\phi}
$$

When normalized, $S_{\phi}$ is the unique autonomous generating function of $\phi$. Any $C^{1}$-small diffeomorphism is engulfable. Let $H^{\phi}$ denote the special Hamiltonian function defined by the equation

$$
\Phi\left(\Delta_{\phi_{H}^{t}}\right)=\text { graph } t d S_{\phi},
$$

where $\Delta_{\phi_{H}^{t}}$ is the graph of $\phi_{H^{\phi}}^{t}$ inside $(M \times M,-\omega \oplus \omega)$. The Hamiltonian function $H^{\phi}$ is quasi-autonomous and so

$$
\int_{0}^{1} \max _{x \in M} H^{\phi}(x, t)-\min _{x \in M} H^{\phi}(x, t) d t=\operatorname{osc}\left(S_{\phi}\right)=\max S_{\phi}-\min S_{\phi} .
$$


Of course when $\phi$ is also engulfable there is the additional inequality

$$
A(\phi ; 1) \leq \gamma(\phi) \leq\|\phi\|_{\text {Hofer }} \leq\left\|H^{\phi}\right\|=\operatorname{osc}\left(S_{\phi}\right) .
$$

In the setting of the periodic Floer homology there are thick and thin decompositions of the Floer boundary map and pants product [21, Propositions 9.1 and 9.3] and in the case of $C^{1}$-small diffeomorphisms, these thick and thin decompositions yield the existence of a thin curve $u$ satisfying equation (1.7) with

$$
\left\{\begin{array}{l}
u_{1}(-\infty)=\left[x^{-}, \widetilde{x^{-}}\right], u_{N}(+\infty)=\left[x^{+}, \widetilde{x^{+}}\right] \\
E_{J}(u)=\operatorname{osc}\left(S_{\phi}\right)
\end{array}\right.
$$

which in turn, after some further analysis, implies $A(\phi ; 1) \geq \operatorname{osc}\left(S_{\phi}\right)$. As a result

$$
A(\phi ; 1)=\gamma(\phi)=\|\phi\|_{\text {Hofer }}=\operatorname{osc}\left(S_{\phi}\right),
$$

see [21, Theorem F and Proposition 9.6].

However, when the diffeomorphism $\phi$ is engulfable, the thick and thin approach does not directly apply and so we must use Theorem A to produce the existence of a local curve $u$ satisfying (1.7) and (1.10), see Proposition 4.2. This is the main step in the proof of

Theorem B. Let $\phi$ be a non-degenerate, engulfable Hamiltonian diffeomorphism and assume that the oscillation of its generating function $S_{\phi}$ satisfies

$$
\operatorname{osc}\left(S_{\phi}\right)<\frac{\sigma(M, \omega)}{2} .
$$

For such a diffeomorphism, the following string of equalities holds.

$$
A(\phi ; 1)=\gamma(\phi)=\|\phi\|_{\text {Hofer }}=\left\|H^{\phi}\right\|=\operatorname{osc}\left(S_{\phi}\right) .
$$

It is not known whether the homological area depends continuously on the Hamiltonian $\phi$ and so Theorem B remains open for degenerate $\phi$. Nevertheless, Theorem B is sufficient to prove

Corollary C. Let $\phi$ be any engulfable Hamiltonian, possibly degenerate, whose generating function $S_{\phi}$ satisfies

$$
\operatorname{osc}\left(S_{\phi}\right)<\frac{\sigma(M, \omega)}{2} .
$$

The path $t \rightarrow \phi_{H^{\phi}}^{t}$ is Hofer and spectral length minimizing. In fact

$$
\gamma(\phi)=\|\phi\|_{\text {Hofer }}=\left\|H^{\phi}\right\|=\operatorname{osc}\left(S_{\phi}\right) .
$$

Corollary D. The collection $\mathcal{H}$ of all engulfable Hamiltonians satisfying Condition (1.11)

$$
\mathcal{H} \subset \operatorname{Ham}(M, \omega)
$$

is an open, path-connected neighborhood of the identity in the $C^{1}$-topology such that any element of $\mathcal{H}$ admits a Hofer and spectral length minimizing 
path to the identity. The neighborhood $\mathcal{H}$ with either the Hofer or spectral norm is isometric to the normed vector space $C_{m}^{\infty}(M)$ of mean zero functions $f: M \rightarrow \mathbb{R}$ with the norm given by the oscillation, osc $(f)=\max f-\min f$.

For $C^{1}$-small Hamiltonians Lalonde and McDuff [10] and McDuff [12] obtain this result under the Hofer metric, and Oh [21] for both the Hofer and spectral distances.

Suppose the symplectic manifold is symplectically aspherical. In this case, hypothesis (1.11) is not restrictive since there are no non-constant pseudo-holomorphic spheres and $\sigma(M, \omega)=+\infty$. It is also worth comparing Corollary $\mathrm{C}$ to Ostrover's result Theorem $1.2[\mathbf{2 2}]$ that when $(M, \omega)$ is symplectically aspherical, there exist Hamiltonian diffeomorphisms (far from the identity) admitting no Hofer length minimizing path to the identity. An example of this kind is also given on $S^{2}$ by Lalonde and McDuff [10].

An explicit description of the size of the neighborhood, $\mathcal{N} \subset \operatorname{Ham}(M, \omega)$, McDuff constructs is explained in [12, Remark 3.5]. It appears that this description requires some control on both the Hofer length and $C^{2}$-norm of the generating Hamiltonian function, which we avoid.

In some cases, the Darboux neighborhood of the diagonal $\mathcal{U} \subset M \times M$ can be quite large. One may ask if there is a relationship between the diameter of the Hamiltonian diffeomorphism group and the size of the Darboux neighborhood of the diagonal.

\section{2. $\lambda$-Homotopy}

Chekanov [2] defines an algebraic framework to prove an existence theorem of Lagrangian intersections. We recall the definitions and prove a new algebraic result, Proposition 2.3, needed in the proof of Theorem B.

Let $\Gamma$ be a free abelian group and $\lambda: \Gamma \rightarrow \mathbb{R}$ be an injective homomorphism. Such a mapping is called a weight function. Denote

$$
\Gamma^{+}=\{g \in \Gamma \mid \lambda(g)>0\}, \quad \Gamma^{-}=\{g \in \Gamma \mid \lambda(g)<0\} .
$$

Let $k$ be a commutative ring with non-zero unity and $K=k(\Gamma)$ be the group ring over $k$. The group ring $K$ includes the group $\Gamma$ as a subgroup.

Now let $M$ be a $k$-module. The group $\Gamma$ need not act on $M$, but $\Gamma$ does act on $M \otimes K$ via the second factor $g(v \otimes \bar{g}):=v \otimes g(\bar{g})$. Furthermore, $M \otimes K$ admits the decomposition

$$
M \otimes K=M^{+} \oplus M^{0} \oplus M^{-},
$$

where $M^{+}=\Gamma^{+}(M \otimes K), M^{0}=M$ and $M^{-}=\Gamma^{-}(M \otimes K)$.

The above splitting supports two projections

$$
p^{+}: M \otimes K \longrightarrow M^{+} \oplus M^{0} \quad \text { and } \quad p^{-}: M \otimes K \longrightarrow M^{0} \oplus M^{-},
$$


$p^{+}(v \otimes \bar{g})=v \otimes \sum p^{+}\left(\alpha_{i}\right) g_{i}$, where

$$
p^{+}\left(\alpha_{i}\right)= \begin{cases}0 & \text { if } \lambda\left(g_{i}\right)<0 \\ \alpha_{i} & \text { if } \lambda\left(g_{i}\right) \geq 0\end{cases}
$$

and similarly for $p^{-}$.

Now assume in addition that $(M, \partial)$ is a differential $k$-module with $\partial \circ \partial=0$. Notice that $\partial$ extends to $M \otimes K$ by setting $\partial(v \otimes \bar{g})=(\partial v) \otimes \bar{g}$. Also the differential $\partial$ and the projections $p^{+}, p^{-}$are commuting operators because $\partial$ acts on $M$ while the projections act only on $K$ in $M \otimes K$.

Definition 2.1 (Chekanov [2]). Two $K$-linear maps $\psi_{1}, \psi_{2}: M \otimes K \rightarrow M \otimes K$ are called $\lambda$-homotopic if there exists a $K$-linear map $h: M \otimes K \rightarrow M \otimes K$ such that

$$
p^{+}\left(\psi_{1}-\psi_{2}+h \circ \partial+\partial \circ h\right) p^{-}=0 .
$$

Notice that if $\psi_{1}, \psi_{2}$ were chain homotopic maps in the usual sense, then equation (2.3) holds. The definition implies that $\psi_{1}$ and $\psi_{2}$ are chain maps from negative to positive weight. The essential algebraic lemma, observed first by Chekanov, from which this section really follows is

Lemma 2.2 (Chekanov [2]). Let $V$ be the homology of $(M, \partial)$. Set $W=$ $V \otimes K$. For any non-zero $r \in W$, there exists $g \in \Gamma$ for which $g \cdot r=q^{0}+q^{-}$, with $q^{0} \in V \subset M^{0}$ non-zero and $q^{-} \in M^{-}$.

Proof. We have $r=v \otimes \bar{g} \in W=V \otimes K$, with $\bar{g}=\sum \alpha_{i} g_{i}, \alpha_{i} \in k, g_{i} \in \Gamma$. Let $g=g_{j} \in \Gamma$ be such that

$$
g=g_{\max }^{-1} \text { with } \lambda\left(g_{\max }\right)=\max _{\alpha_{i} \neq 0} \lambda\left(g_{i}\right) .
$$

The quantity $\max _{\alpha_{i} \neq 0} \lambda\left(g_{i}\right)$ is called the valuation of $g$. Then by the action of $\Gamma$ on $K$

$$
g(r)=v \otimes \sum_{g_{n} g_{j}=g_{i}} \alpha_{n} g_{i} .
$$

Finally if $\alpha_{n} \neq 0$, then $\lambda\left(g_{i}\right)=\lambda\left(g_{n}\right)+\lambda\left(g_{j}\right) \leq-\lambda\left(g_{j}\right)+\lambda\left(g_{j}\right)=0$ and hence $\lambda\left(g_{i}\right) \leq 0$.

What follows below is the main algebraic result of the current paper. An isomorphism on homology cannot be $\lambda$-homotopic to the zero map provided the isomorphism commutes with the projection $p^{+}$.

Proposition 2.3. Suppose $(M, \partial)$ is a differential $k$-module with nontrivial homology. Suppose the linear maps $\psi_{1}, \psi_{2}: M \otimes K \rightarrow M \otimes K$ are $\lambda$-homotopic. If $\psi_{1}$ descends to an isomorphism $\psi_{1}: H(M, \partial) \stackrel{\cong}{\rightarrow} H(M, \partial)$ and the induced map commutes with $p^{+}$on homology (i.e., up to a boundary on the chain level), then $\psi_{2} \neq 0$. 
Proof. Choose a submodule of $\partial$-cycles $V \subset M=M^{0}$ representing the homology $H(M, \partial)$. Let $W=V \otimes K$. Note that $W$ is not trivial. By Lemma 2.2 for any non-zero $r \in W$ there exists $g \in \Gamma$ for which $g \cdot r=q^{0}+q^{-}$, with $q^{0} \in V \subset M^{0}$ non-zero and $q^{-} \in M^{-}$.

To prove the proposition suppose on the contrary that $\psi_{2}=0$. Applying equation (2.3) to $q^{0}+q^{-}$, we see

$$
\begin{aligned}
0 & =p^{+}\left(\psi_{1}-\psi_{2}+h \circ \partial+\partial \circ h\right) p^{-}\left(q^{0}+q^{-}\right) \\
& =p^{+}\left(\psi_{1}-\psi_{2}+h \circ \partial+\partial \circ h\right)\left(q^{0}+q^{-}\right) \\
& =p^{+}\left(\psi_{1}\left(q^{0}+q^{-}\right)\right)+p^{+}(h \circ \partial+\partial \circ h)\left(q^{0}+q^{-}\right) \\
& =p^{+}\left(\psi_{1}\left(q^{0}+q^{-}\right)\right)+\partial\left(p^{+} h\left(q^{0}+q^{-}\right)\right) .
\end{aligned}
$$

Here we have used the linearity of all the maps that $q^{0}+q^{-}$is a cycle and that the projection $p^{+}$commutes with the boundary. By assumption, $p^{+}$and $\psi_{1}$ commute up to a boundary, say $\partial c$, and so continuing

$$
\begin{aligned}
0 & =\psi_{1}\left(q^{0}\right)+\partial c+\partial\left(p^{+} h\left(q^{0}+q^{-}\right)\right) \\
& =\psi_{1}\left(q^{0}\right)+\partial\left(c+p^{+} h\left(q^{0}+q^{-}\right)\right)
\end{aligned}
$$

which implies that $\psi_{1}\left(q^{0}\right)$ is a boundary. Hence, $\psi_{1}$ cannot be an isomorphism, a contradiction.

Remark 2.4. The downward Novikov ring $\Lambda^{\downarrow}$ is defined to be

$$
\Lambda^{\downarrow}=\left\{\sum_{i} \alpha_{i} g_{i} \mid(\forall) t \in \mathbb{R} \#\left\{g_{i} \mid \alpha_{i} \neq 0 \text { and } \lambda\left(g_{i}\right)>t\right\}<\infty\right\} .
$$

Because the valuation of any element in the downward Novikov ring is finite, Lemma 2.2 continues to hold with the group ring $K$ replaced with $\Lambda^{\downarrow}$. In other words, the notion of $\lambda$-homotopy extends to this coefficient ring.

\section{The Proof of Theorem A}

Let $L$ be a closed, connected $n$-dimensional Lagrangian submanifold of the tame symplectic $(P, \omega)$. Let $\mathcal{U}$ be a Darboux-Weinstein neighborhood of $L$. Fix a reference path $l_{0}$ on $L$. Given a (normalized) time-dependent Hamiltonian function $H: P \times[0,1] \rightarrow \mathbb{R}$, the corresponding action functional $\mathcal{A}_{H}$ is real valued on the Novikov covering $\widetilde{\Omega}_{0}\left(L, l_{0} ; P, \omega\right)=\widetilde{\Omega}_{0} \stackrel{\pi}{\rightarrow} \Omega_{0}$ of the space $\Omega_{0}$ of contractible paths beginning and ending on $L$. $\widetilde{\Omega}_{0}$ consists of equivalence classes $\ell$ where the half-disk $w$ provides the homotopy to $l_{0}$ and two pairs $\left(l_{1}, \widetilde{l_{1}}\right)$ and $\left(l_{2}, \widetilde{l_{2}}\right)$ are equivalent if $l_{1}=l_{2}$ and the Maslov index $\mu_{L}$ and symplectic action $I_{\omega}$ homomorphisms vanish on the glued disk $\widetilde{l_{1}} \# \widetilde{\widetilde{l_{2}}}$ (Figure 1). The Maslov index conventions and the Novikov covering are discussed in greater detail in [5, Chapter 2, Sections 2 and 3]. 


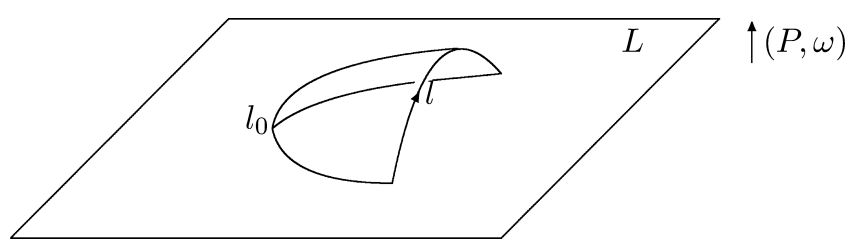

Figure 1. Half-disk $\widetilde{l}$ with boundary on $L$.

The choice of sign is

$$
\mathcal{A}_{H}(\ell):=-\int \widetilde{l}^{*} \omega-\int_{0}^{1} H(l(t), t) d t
$$

Denote $\Gamma=\Gamma\left(L, l_{0} ; P, \omega\right)$ the automorphism group of this cover, which is a free abelian group

$$
\Gamma=\frac{\pi_{2}(P, L)}{\operatorname{ker}\left(\mu_{L}\right) \cap \operatorname{ker}\left(I_{\omega}\right)} .
$$

$\Gamma$ acts by gluing a disk: $g \cdot \ell=[l, \widetilde{l} \# g]$. The symplectic action provides the weight homomorphism

$$
\lambda(g):=-\int g^{*} \omega
$$

Let $\sigma(L ; P, \omega)$ be the minimal area of a pseudo-holomorphic sphere or disk with boundary on $L$ in $P$ and assume for the remainder of this section that the Hofer length of $H$ satisfies $\|H\|<\sigma / 2$. Let $0<s \ll 1$ and fix the constant $\delta>0$ to satisfy $\|H\|<\delta / 2<\sigma / 2$.

For the parameterized Hamiltonian $s H$, denote the critical points of the action functional $\mathcal{A}_{s H}$

$$
\operatorname{Crit}\left(\mathcal{A}_{s H}\right)=C F(L, s H)=C F(s)
$$

and the vector space of Floer chains

$$
C F_{*}(s)=C F_{*}\left(L, s H ; \mathbb{Z}_{2}\right)=\operatorname{span}_{\mathbb{Z}_{2}}(C F(s)) .
$$

We will make a number of assumptions on the size of the parameter $s$ in the course of the proof of Theorem A; however, this parameter does not appear in the statement of Theorem B. The first of these conditions is that

$$
\phi_{s H}^{t}(L) \subset \mathcal{U} \quad(\forall) t \in[0,1] .
$$

Two elements of $C F(s)$ are said to be equivalent (see [2, section 4]) if they belong to the same connected component of the set

$$
\pi^{-1}\left\{l \in \Omega_{0}: l([0,1]) \subset \mathcal{U}\right\} \subset \widetilde{\Omega}_{0} .
$$

The first assumption on the parameter $s$ guarantees that the set of equivalence classes is not empty. Fixing any equivalence class $C F^{\circ}(s)$ yields the decomposition $C F_{*}(s)=C F_{*}^{\circ}(s) \otimes K$, where $C F_{*}^{\circ}(s)=\operatorname{span}_{\mathbb{Z}_{2}} C F^{\circ}(s)$ and 
$K=\mathbb{Z}_{2}(\Gamma)$ denotes the group ring. Observe that for two equivalent critical points $[x, \tilde{x}],[z, \tilde{z}] \in C F^{\circ}(s)$ there is a topological (half) cylinder $C$ with boundary on $L$ contained in $\mathcal{U}$ such that $\widetilde{x} \# C \simeq \widetilde{z}$. Hence $\widetilde{x}$ and $\widetilde{z}$ have equal symplectic area. Thus for equivalent critical points

$$
\mathcal{A}_{s H}([x, \tilde{x}])-\mathcal{A}_{s H}([z, \tilde{z}])=s\left(\int H(z(t), t)-H(x(t), t) d t\right),
$$

and so if we assume further that $s<\delta /(2|| H \|+1)$, we will have

$$
[x, \tilde{x}],[z, \tilde{z}] \in C F^{\circ}(s) \Longrightarrow \mathcal{A}_{s H}([x, \tilde{x}])-\mathcal{A}_{s H}([z, \tilde{z}])<\frac{\delta}{2} .
$$

The sets of positive and negative weight critical points are denoted

$$
C F^{+}(L, s H)=\Gamma^{+}\left(C F^{\circ}\right) \text { and } C F^{-}(L, s H)=\Gamma^{-}\left(C F^{\circ}\right) .
$$

Let $J$ be a (possibly time-dependent) almost complex structure compatible to $\omega$. Again the Floer differential is defined by counting finite energy solutions of equation (1.2). The full Floer moduli space of finite energy solutions $\mathcal{N}(L, s H ; J, \omega)=\mathcal{N}(s)$ ranges over all critical points of $\mathcal{A}_{s H}$. The finite energy solutions converge exponentially as $\tau \rightarrow \pm \infty$ to paths $x^{ \pm}:[0,1] \rightarrow P$ satisfying equation (1.1).

For generic $s, \mathcal{N}([x, \tilde{x}],[z, \tilde{z}] ; s)$ is a smooth manifold of dimension $\mu_{L}([x, \tilde{x}])-\mu_{L}([z, \tilde{z}])$, when it is not empty, see $[\mathbf{6}]$.

The length of a Floer trajectory $u \in \mathcal{N}([x, \tilde{x}],[z, \tilde{z}], s)$ is defined to be the action difference at its ends

$$
\mathcal{L}(u):=\mathcal{A}_{s H}([x, \tilde{x}])-\mathcal{A}_{s H}([z, \tilde{z}]) .
$$

We say that $u$ is somewhat thin if $\mathcal{L}(u) \leq\|H\|+\delta$, thin if $\mathcal{L}(u) \leq \delta$ and local if image $(u) \subset \mathcal{U}$. Curves which exit the Darboux-Weinstein neighborhood are called thick. A consequence of Gromov's compactness theorem is Oh's thick and thin decomposition theorem that implies when $s$ is sufficiently small and $J$ is $C^{0}$-close to a time-independent compatible almost complex structure that every somewhat thin trajectory is thin and every (somewhat) thin trajectory is local (see [2, Lemma 6] and [13, Proposition 4.1]). Our third assumption on the size of the parameter $s$ is that it is small enough to ensure the thick and thin decomposition. We denote the collection of thin trajectories $\mathcal{N}^{\circ}(L, s H)$ and local trajectories $\mathcal{N}^{\mathcal{U}}(L, s H)$. After dividing by the time-shift, the thin Floer differential $\partial^{\circ}$ is defined by $\mathcal{N}^{\circ} / \mathbb{R}:=\widehat{\mathcal{N}}^{\circ}(L, s H)$

$$
\partial^{\circ}([x, \tilde{x}])=\sum_{\mu_{L}([x, \tilde{x}])-\mu_{L}([z, \tilde{z}])=1} \#_{\mathbb{Z}_{2}} \widehat{\mathcal{N}}^{\circ}([x, \tilde{x}],[z, \tilde{z}], s)[z, \tilde{z}] .
$$

When $s$ is sufficiently small and the almost complex structure is close to an autonomous almost complex structure, the thin differential satisfies 
$\partial^{\circ} \circ \partial^{\circ}=0$ and the resulting homology is isomorphic to the singular homology of $L$

$$
H\left(C F_{*}(s), \partial^{\circ}\right):=H F_{*}^{\circ}\left(L, s H ; \mathbb{Z}_{2}\right) \cong H_{*}\left(L ; \mathbb{Z}_{2}\right),
$$

see [2, Lemma 7] and [13, Theorem 4.7].

The thin Floer cap product $[L] \cap_{s}^{\circ}(\cdot): C F_{*}^{\circ}(s) \rightarrow C F_{*}^{\circ}(s)$ is defined by counting elements $u \in \mathcal{N}^{\circ}(L, s H)$ passing through a generic point $p \in L$.

$$
\begin{aligned}
& \mathcal{N}^{\circ}(L, s H ; p)=\left\{u \in \mathcal{N}^{\circ} \mid u(0,0)=p\right\} \\
& {[L] \cap_{s}^{\circ}([x, \tilde{x}])=\sum_{\mu_{L}([x, \tilde{x}])-\mu_{L}([z, \tilde{z}])=n} \#_{\mathbb{Z}_{2}} \mathcal{N}^{\circ}([x, \tilde{x}],[z, \tilde{z}], s ; p)[z, \tilde{z}] .}
\end{aligned}
$$

The collection of such maps is denoted $\mathcal{N}^{\circ}(L, s H ; p)$ (or $\mathcal{N}^{\circ}([x, \tilde{x}]$, $[z, \tilde{z}], s ; p)$ or $\mathcal{N}^{\mathcal{U}}(L, s H ; p)$, etc. $)$. Because of the pointed condition $u(0,0)=$ $p$, the dimension is reduced by $n$, the dimension of $L$. Again the thick and thin decomposition holds.

Proposition 3.1 ([13, Proposition 4.1] and [2, Lemma 6]). Fix a timeindependent compatible almost complex structure $J_{0}$ on $P$. There exists $s^{\prime}>0$ with the property that for any $0<s<s^{\prime}$ if $\left\|J-J_{0}\right\|_{C^{0}(P \times[0,1])}<s$, then any somewhat thin cap trajectory is thin and any (somewhat) thin cap trajectory is local.

Proposition 3.1 implies that the moduli space $\mathcal{N}^{\circ}(L, s H ; p)$ is isolated, say by the length defined above, within $\mathcal{N}(L, s H ; p)$. Passing to the cotangent bundle, the work of $[\mathbf{3}, \mathbf{4}, \mathbf{1 5}]$ implies that $[L] \cap_{s}^{\circ}(\cdot)$ descends to an isomorphism on $H F_{*}^{\circ}\left(L, s H ; \mathbb{Z}_{2}\right)$. (In particular A. Floer originally proved the injectivity of the cap action.) The next assumption on $s$ is that is it small enough to ensure

$$
[L] \cap_{s}^{\circ}(\cdot): H F_{*}^{\circ}\left(L, s H ; \mathbb{Z}_{2}\right) \stackrel{\cong}{\longrightarrow} H F_{*-n}^{\circ}\left(L, s H ; \mathbb{Z}_{2}\right) .
$$

The thin cap product shifts the grading by $n$, the dimension of the Lagrangian $L$.

Setting the parameter $s=1$, the local cap product $[L] \cap^{\mathcal{U}}(\cdot)$ is defined by the local curves in the moduli space $\mathcal{N}^{\mathcal{U}}(L, H ; p):=\{v \in \mathcal{N}(L, 1$. $H ; p) \mid \operatorname{Image}(u) \subset \mathcal{U}\}$.

$$
[L] \cap^{\mathcal{U}}([y, \tilde{y}])=\sum_{\mu_{L}([y, \tilde{y}])-\mu_{L}\left(\left[y^{\prime}, \tilde{y}^{\prime}\right]\right)=n} \#_{\mathbb{Z}_{2}} \mathcal{N}^{\mathcal{U}}\left([y, \tilde{y}],\left[y^{\prime}, \tilde{y^{\prime}}\right] ; p\right)\left[y^{\prime}, \tilde{y}^{\prime}\right] .
$$

Within the Darboux neighborhood of $L$, bubbling-off cannot occur and so $[L] \cap^{\mathcal{U}}(\cdot)$ is defined; however, this map need not agree with the singular cap product. We have thus far fallen short in proving that the moduli space of local curves is isolated among all curves. This fact is what motivated the use of $\lambda$-homotopy. For the same reason, when $s=1$, the local Floer homology might not be defined. In particular $\partial^{\mathcal{U}}$ need not square to zero.

The continuation maps $\Phi^{+}$and $\Phi^{-}$are constructed by introducing monotone continuation functions $\rho^{+}$and $\rho^{-}$of $\tau$ and a non-autonomous 
Floer equation. We sketch definition of the map $\Phi^{+}$, referring the reader to [2] for the full details. Let $J^{\rho^{+}}$be a $\tau$ (and $t$ ) dependent almost complex structure such that it is independent of $\tau$, when $|\tau| \gg 0$.

Let $b_{+}(H)=\int_{0}^{1} \max _{x} H(x, t) d t$ and $b_{-}(H)=-\int_{0}^{1} \min _{x} H(x, t) d t$. Let $\rho^{+}$be a smooth increasing function of $\tau$ so that $\rho^{+}(\tau)=1$ for $\tau \ll 1$ and $\rho^{+}(\tau)=s$ for $\tau \ll-1$. Consider the continuation equation

$$
\left\{\begin{array}{l}
\frac{\partial u}{\partial \tau}+J^{\rho^{+}}(u, t)\left(\frac{\partial u}{\partial t}-X_{\rho^{+} H}(u, t)\right)=0 \\
u: \mathbb{R} \times[0,1] \rightarrow P \\
u(\tau, 0), u(\tau, 1) \subset L
\end{array}\right.
$$

and define

$$
\mathcal{N}_{\rho^{+}}=\left\{u: \mathbb{R} \times[0,1] \rightarrow P \mid E_{J}(u)<+\infty \text { and } u \text { satisfies }(3.6)\right\}
$$

The length of a continuation trajectory $u^{+} \in \mathcal{N}_{\rho^{+}}$can be defined as before (and could be negative) $u^{+} \in \mathcal{N}_{\rho^{+}}$

$$
\mathcal{L}\left(u^{+}\right)=\mathcal{A}_{s H}([x, \tilde{x}])-\mathcal{A}_{H}([y, \tilde{y}]),
$$

where $u^{+}(-\infty)=[x, \tilde{x}] \in C F(s)$ and $u^{+}(+\infty)=[y, \tilde{y}] \in C F(1)$. The continuation trajectories $u^{+}, u^{-}$are called thin if

$$
\left\{\begin{array}{l}
\mathcal{L}\left(u^{+}\right) \leq(1-s) \cdot b_{+}(H)+\delta \\
\mathcal{L}\left(u^{-}\right) \leq(1-s) \cdot b_{-}(H)+\delta
\end{array}\right.
$$

and the collection of all thin continuation trajectories we denote $\mathcal{N}_{\rho^{+}}^{\circ}$ and $\mathcal{N}_{\rho^{-}}^{\circ}$. Observe that to be thin, a continuation trajectory satisfies a weaker inequality than is required for a Floer trajectory to be thin.

Lemma 3.2. [2, Lemma 9] If $u^{+} \in \mathcal{N}_{\rho^{+}}$, then $\mathcal{L}\left(u^{+}\right) \geq-(1-s) \cdot b_{-}(H)$. If $u^{-} \in \mathcal{N}_{\rho^{-}}$, then $\mathcal{L}\left(u^{-}\right) \geq-(1-s) \cdot b_{+}(H)$. For the thin continuation trajectories, $E\left(u^{ \pm}\right) \leq\|H\|+\delta$.

Consequently, the continuation map $\Phi^{+}: C F(s) \rightarrow C F(1)$ defined by

$$
\Phi^{+}([x, \tilde{x}])=\sum_{\mu_{L}([x, \tilde{x}])-\mu_{L}([y, \tilde{y}])=0} \#_{\mathbb{Z}_{2}} \mathcal{N}_{\rho^{+}}^{\circ}([x, \tilde{x}],[y, \tilde{y}])[y, \tilde{y}]
$$

is finite. Again we refer to [2, proof of Lemma 9] for the details of the finiteness argument. Notice that a thick and thin decomposition is not needed to make this definition. 
The important point is that these linear maps provide a bridge from the Floer complexes corresponding to $0<s \ll 1$ and $s=1$,

$$
\Phi^{+}: C F_{*}(L, s H) \longrightarrow C F_{*}(L, H) \quad \text { and } \quad \Phi^{-}: C F_{*}(L, H) \longrightarrow C F_{*}(L, s H) .
$$

The main result in [2] is that when $s$ is sufficiently small and $\|H\|<\sigma$, there exists a $\lambda$-homotopy $h$ from the identity to the composition $\Phi^{-} \circ \Phi^{+}$. As a consequence, the displacement energy of any Lagrangian submanifold is larger than $\sigma$. Our $\lambda$-homotopy is defined similarly, where we must also take into consideration the marked point $p \in L$. We now provide the details.

Let $R \in[s,+\infty)$ and $\mu_{R}: \mathbb{R} \rightarrow[s, 1]$ be a smooth function with all the following properties. First, $0 \leq \mu_{R}^{\prime}(\tau)$ for $\tau \leq 0$ and $\mu_{R}^{\prime}(\tau) \leq 0$ for $\tau \geq 0$ and for $R \geq 1$

$$
\mu_{R}(\tau)=\left\{\begin{array}{l}
1, \quad-R \leq \tau \leq R \\
s, \quad|\tau| \geq R+1
\end{array}\right.
$$

while when $s \leq R \leq 1, \mu_{R}=R \cdot \mu_{1}$. Let $J^{\mu_{R}}$ be a $\tau$-dependent almost complex structure that is $\tau$-independent for $\tau \ll 0$. The $\lambda$-homotopy is defined via the finite energy solutions of the non-autonomous Floer equation

$$
\left\{\begin{array}{l}
\frac{\partial u}{\partial \tau}+J^{\mu_{R}}(u, t)\left(\frac{\partial u}{\partial t}-X_{\mu_{R}(\tau) H}(u, t)\right)=0 \\
u(\tau, 0), u(\tau, 1) \subset L \\
u(0,0)=p \in L
\end{array}\right.
$$

The collection of all finite energy solutions is denoted $\mathcal{N}_{\mu_{R}}=\mathcal{N}_{\mu_{R}}(L, s H ; p)$. The parameterized moduli space is defined to be $\mathcal{N}_{\mu}=\{(R, v): v \in$ $\left.\mathcal{N}_{\mu_{R}}(L, s H ; p)\right\}$ and by the parameterized index theorem (see [14]), the local dimension of $\mathcal{N}_{\mu}$ is one greater than the difference of Maslov indices of the asymptotic limits and so defines a map of degree +1 . The formula for the pointed $\lambda$-homotopy $h^{p}: C F(s) \rightarrow C F(s)$ on generators is

$$
h^{p}([x, \tilde{x}])=\sum_{[y, \tilde{y}] \in C F^{\circ} \cup C F^{+}} \#_{\mathbb{Z}_{2}}\left\{\mathcal{N}_{\mu}([x, \tilde{x}],[y, \tilde{y}] ; p)\right\}[y, \tilde{y}],
$$

where $[x, \tilde{x}] \in C F^{-} \cup C F^{\circ}$. Because the energy is controlled by the Hofer norm, bubbling-off does not occur and so the sum is finite. The induced map is linear over $K=\mathbb{Z}_{2}(\Gamma)$.

We now verify the $\lambda$-homotopy equation.

$$
p^{+}\left([L] \cap_{s}^{\circ}(\cdot)-\Phi^{-} \circ[L] \cap^{\mathcal{U}}(\cdot) \circ \Phi^{+}+h^{p} \circ \partial^{\circ}+\partial^{\circ} \circ h^{p}\right) p^{-}=0
$$

for all $[x, \tilde{x}] \in C F^{\circ}(s)$. We write the left hand side of equation (3.12) as

$$
\sum \#_{\mathbb{Z}_{2}} \mathcal{S}([x, \tilde{x}],[z, \tilde{z}])[z, \tilde{z}]
$$


where $\mathcal{S}([x, \tilde{x}],[z, \tilde{z}])$ is the union of the following four sets. For the remainder of this section, each of $[x, \tilde{x}] \in C F^{\circ}(s) \cup C F^{-}(s),[z, \tilde{z}] \in$ $C F^{+}(s) \cup C F^{\circ}(s)$ and $[y, \tilde{y}],\left[y^{\prime}, \tilde{y}^{\prime}\right] \in C F(1)$ represent generic critical points of the respective action functionals.

(1) A thin cap trajectory $u \in \mathcal{N}^{\circ}([x, \tilde{x}],[z, \tilde{z}], s ; p)$.

(2) A triple $\left(u^{-}, v, u^{+}\right)$with $u^{+} \in \mathcal{N}_{\rho^{+}}^{\circ}([x, \tilde{x}],[y, \tilde{y}]), v \in \mathcal{N}^{\mathcal{U}}\left([y, \tilde{y}],\left[y^{\prime}, \tilde{y^{\prime}}\right] ; p\right)$ and $u^{-} \in \mathcal{N}_{\rho^{-}}^{\circ}\left(\left[y^{\prime}, \tilde{y^{\prime}}\right],[z, \tilde{z}]\right)$.

(3) A thin unparameterized gradient trajectory $u \in \widehat{\mathcal{N}}^{\circ}([x, \tilde{x}],[z, \tilde{z}], s)$ and element $(R, v)$ with $v \in \mathcal{N}_{\mu_{R}}([x, \tilde{x}],[z, \tilde{z}] ; p)$.

(4) An element $(R, v)$ with $v \in \mathcal{N}_{\mu_{R}}([x, \tilde{x}],[z, \tilde{z}] ; p)$ and a thin unparameterized gradient trajectory $u \in \widehat{\mathcal{N}}^{\circ}([x, \tilde{x}],[z, \tilde{z}], s)$.

By the Floer gluing theorem, each term above lies at the end of the one-dimensional portion $\mathcal{Q}$ of $\mathcal{S}$. In fact $\mathcal{Q}$ is compact and the correspondence is one-one. The compactness of $\mathcal{Q}$ follows from the uniform energy estimate which is a consequence of the assumption $\|H\|_{\text {Hofer }}<\sigma / 2$ (see [2, Lemma 10] and [14, Lemma 2.2]).

On the other hand, by the Gromov-Floer compactness theorem (see [25, Lemma 6.2]) every end of $\mathcal{Q}$ may be compactified by one of the following.

(1) A cap trajectory $u \in \mathcal{N}^{\circ}([x, \tilde{x}],[z, \tilde{z}], s ; p)$.

(2) A triple $\left(u^{-}, v, u^{+}\right)$with $u^{+} \in \mathcal{N}_{\rho^{+}}([x, \tilde{x}],[y, \tilde{y}]), v \in \mathcal{N}^{\mathcal{U}}\left([y, \tilde{y}],\left[y^{\prime}, \tilde{y^{\prime}}\right] ; p\right)$ and $u^{-} \in \mathcal{N}_{\rho^{-}}\left(\left[y^{\prime}, \tilde{y^{\prime}}\right],[z, \tilde{z}]\right)$.

(3) An unparameterized gradient trajectory $u \in \widehat{\mathcal{N}}([x, \tilde{x}],[z, \tilde{z}], s)$ and element $(R, v)$ with $v \in \mathcal{N}_{\mu_{R}}([x, \tilde{x}],[z, \tilde{z}] ; p)$.

(4) An element $(R, v)$ with $v \in \mathcal{N}_{\mu_{R}}([x, \tilde{x}],[z, \tilde{z}] ; p)$ and an unparameterized gradient trajectory $u \in \widehat{\mathcal{N}}([x, \tilde{x}],[z, \tilde{z}], s)$.

We must show that in each of the above cases, the relevant trajectories are thin. In fact, this follows from the conjugation by $p^{+}$and $p^{-}$and that $\|H\|<\sigma / 2$. Moreover, that the limit trajectories are thin in cases (3) and (4) follows from the proof of Proposition 8 in [2]. To economize the exposition, we focus on cases (1) and (2).

Let us begin with (1). The asymptotic limits assure us that $[x, \tilde{x}]=$ $g_{x} \cdot\left[x^{\prime}, \tilde{x^{\prime}}\right]$ and that $[z, \tilde{z}]=g_{z} \cdot\left[z^{\prime}, \tilde{z^{\prime}}\right]$ with

$$
-\int\left(g_{z} \# \tilde{z^{\prime}}\right)^{*} \omega \leq 0 \text { and } \int\left(g_{x} \# \tilde{x^{\prime}}\right)^{*} \omega \leq 0 .
$$

Hence, the length of $u$ satisfies

$$
\begin{aligned}
\mathcal{L}(u) & =\mathcal{A}_{s H}([z, \tilde{z}])-\mathcal{A}_{s H}([x, \tilde{x}]) \\
& =-\int \tilde{z}^{*} \omega-s \int H(z, t) d t+\int \tilde{x}^{*} \omega+s \int H(x, t) d t
\end{aligned}
$$




$$
\begin{aligned}
& =-\int\left(g_{z} \# \tilde{z^{\prime}}\right)^{*} \omega-s \int H(z, t) d t+\int\left(g_{x} \# \tilde{x^{\prime}}\right)^{*} \omega+s \int H(x, t) d t \\
& \leq s\left(\int H(x, t)-H(z, t) d t\right) \\
& \leq s\left(\int \max _{x} H(x, t)-\min _{x} H(x, t) d t\right) \\
& =s\|H\| \\
& <\delta
\end{aligned}
$$

which makes it thin.

Figure 2 illustrates the situation of case (2).

To complete the identification we must prove that each $u^{+}$and $u^{-}$is thin. In other words, we must prove $\mathcal{L}\left(u^{+}\right) \leq(1-s) \cdot b_{+}(H)+\delta$ and $\mathcal{L}\left(u^{-}\right) \leq(1-s) \cdot b_{-}(H)+\delta$. (Remember thin continuation trajectories satisfy equation (3.7).) Toward that end, consider the quantity $\mathcal{L}\left(u^{+}\right)+\mathcal{L}\left(u^{-}\right)$. By definition of length

$$
\begin{aligned}
\mathcal{L}\left(u^{+}\right)+\mathcal{L}\left(u^{-}\right) & =\mathcal{A}_{s H}([x, \tilde{x}])-\mathcal{A}_{H}([y, \tilde{y}])+\mathcal{A}_{H}\left(\left[y^{\prime}, \tilde{y^{\prime}}\right]\right)-\mathcal{A}_{s H}([z, \tilde{z}]) \\
& =\left(\int g_{x}^{*} \omega-s \int H(x, t) d t+\left(\mathcal{A}_{H}\left(\left[y^{\prime}, \tilde{y}^{\prime}\right]\right)-\mathcal{A}_{H}([y, \tilde{y}])\right)\right. \\
& \left.-\int g_{z}^{*} \omega+s \int H(z, t) d t\right) .
\end{aligned}
$$

The asymptotic conditions imply that

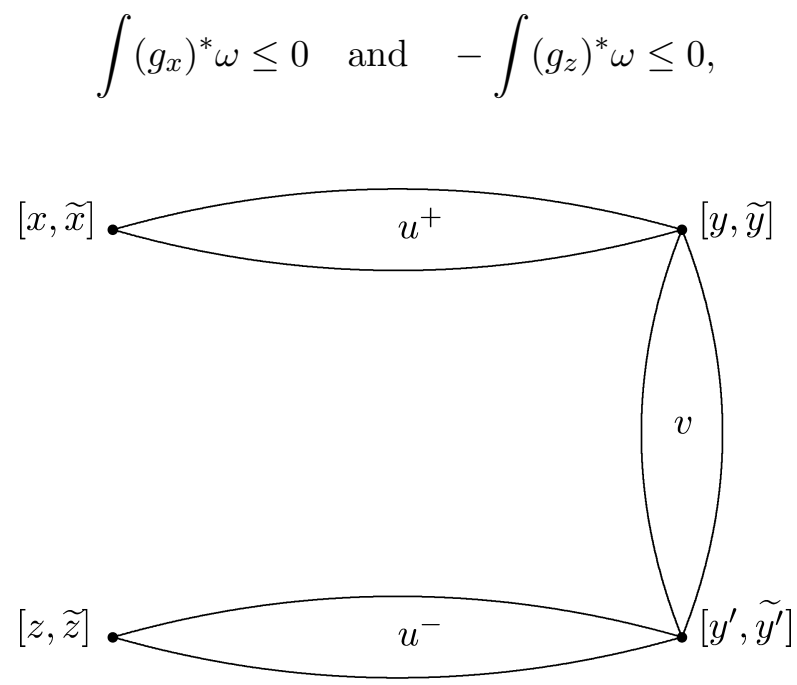

Figure 2. $u^{+}, v, u^{-}$and their respective asymptotic limits. 
therefore (3.13) is smaller than

$$
s\left(\int H(z, t) d t-\int H(x, t) d t\right)+\left(\mathcal{A}_{H}\left(\left[y^{\prime}, \tilde{y^{\prime}}\right]\right)-\mathcal{A}_{H}([y, \tilde{y}])\right) .
$$

Since $v$ is a local cap trajectory from $[y, \tilde{y}]$ to $\left[y^{\prime}, \tilde{y^{\prime}}\right]$, we know that

$$
E(v)=\left(\mathcal{A}_{H}\left(\left[y^{\prime}, \tilde{y^{\prime}}\right]\right)-\mathcal{A}_{H}([y, \tilde{y}])\right),
$$

and furthermore, because $v$ is local, we have $E(v) \leq\|H\|<\delta / 2$. Therefore,

$$
\mathcal{L}\left(u^{+}\right)+\mathcal{L}\left(u^{-}\right) \leq \delta .
$$

Finally it follows that $u^{+}$is thin. Recall from Lemma 3.2 that $\mathcal{L}\left(u^{-}\right) \geq$ $-(1-s) \cdot b_{+}(H)\left(\operatorname{resp} . \mathcal{L}\left(u^{+}\right) \geq-(1-s) \cdot b_{-}(H)\right)$ and so if $u^{+}$were not thin, then

$$
\begin{aligned}
\delta & =-(1-s) \cdot b_{+}(H)+\delta+(1-s) \cdot b_{+}(H) \\
& <\mathcal{L}\left(u^{+}\right)+\mathcal{L}\left(u^{-}\right) \leq \delta
\end{aligned}
$$

a contradiction. Repeating the argument but interchanging $u^{+}$and $u^{-}$ proves that $u^{-}$is also thin. In short, the terms in equation (3.12) are in one-one correspondence with the ends of the compact one-dimensional manifold $\mathcal{Q}$. This implies $h^{p}$ is indeed a $\lambda$-homotopy.

\section{The Proof of Theorem B and Corollary C}

Theorem B is a consequence of Theorem A and Proposition 2.3. Let $(M, \omega)$ be a closed symplectic manifold of dimension $2 n$ and consider the product symplectic manifold $(M \times M,-\omega \oplus \omega)$. The diagonal, $\Delta$, is a Lagrangian submanifold of dimension $2 n=\operatorname{dim}(M)$. In this case $\sigma(\Delta ; M \times M,-\omega \oplus \omega)=$ $\sigma(M ; \omega)$. Fix a Darboux-Weinstein chart $(\Phi, \mathcal{U})$ containing $\Delta$. Let the almost complex structure on $M \times M$ be of the form $\bar{J}=-J_{2 t-1} \oplus J_{1-2 t}$.

Throughout this section consider the Hamiltonian deformation of the diagonal corresponding to the isotopy $t \mapsto$ graph $t d S_{\phi}$, and assume that the Hamiltonian function $H=H^{\phi}$ satisfies $\|H\|=\operatorname{osc}\left(S_{\phi}\right)<\sigma(M, \omega) / 2$. Recall that the Hamiltonian function $H^{\phi}$ is defined by the equation

$$
\Phi\left(\Delta_{\phi_{H^{\phi}}^{t}}\right)=\text { graph } t d S_{\phi}
$$

and that the Hamiltonian vector field of $H$ has only fixed periodic orbits

$$
\operatorname{Per}\left(X_{H}\right) \longleftrightarrow \Delta \cap \Delta_{\phi}
$$

For any non-degenerate Hamiltonian diffeomorphism we have $A(\phi ; 1) \leq$ $\gamma(\phi) \leq\|\phi\|_{\text {Hofer }} \leq\|H\|=\operatorname{osc}\left(S_{\phi}\right)$. Examination of Oh's proof in the $C^{1}$-small case makes clear that the crucial step of the proof is to produce a Floer trajectory connecting the maximum and minimum of the quasiautonomous Hamiltonian $H$. The trajectory we find is in fact local. A curve 
in $M$ is called local if it is in the image of the association of Lemma 4.1 Once this is established we go on to $\operatorname{show} \operatorname{osc}\left(S_{\phi}\right) \leq A(\phi ; 1)$, completing the proof.

Let $F=F^{\phi}:(M \times M) \times[0,1] \rightarrow \mathbb{R}$ be the Hamiltonian function corresponding to $H^{\phi}, F^{\phi}(p, q, t)=H^{\phi}(q, t)$. Set $y^{ \pm}=\left(x^{ \pm}, x^{ \pm}\right) \in M \times M$.

Lemma 4.1. There is an energy preserving one-one correspondence between the moduli spaces $\mathcal{N}^{\mathcal{U}}\left(\Delta, F^{\phi} ; \bar{J} ;(q, q)\right)$ and $\mathcal{M}^{\text {local }}\left(H^{\phi} ; J ; q\right)$.

Proof. By definition, any curve $v \in \mathcal{N}^{\mathcal{U}}\left(\Delta, F^{\phi} ; \bar{J} ;(q, q)\right)$ with

$$
\lim _{\tau \rightarrow \pm \infty} v(\tau, \cdot)=\left[y^{ \pm}, \widetilde{y^{ \pm}}\right] \text {and } \widetilde{y^{-}} \# v \simeq \widetilde{y^{+}}
$$

satisfies

$$
\left\{\begin{array}{l}
\frac{\partial v}{\partial \tau}+\bar{J}(v, t)\left(\frac{\partial v}{\partial t}-X_{F}(v)\right)=0 \\
v: \mathbb{R} \times[0,1] \rightarrow M \times M \\
v(\tau, 0), v(\tau, 1) \subseteq \Delta \\
v(0,0)=(q, q) \in \Delta, \\
\text { image }(v) \subset \mathcal{U}
\end{array}\right.
$$

Integration by parts yields $E_{\bar{J}}(v)=\operatorname{osc}\left(S_{\phi}\right)$. Indeed

$$
\begin{aligned}
E_{\bar{J}}(v) & =\int_{-\infty}^{+\infty}\left|\frac{\partial v}{\partial \tau}\right|^{2} d \tau \\
& =\int v^{*}(-\omega \oplus \omega)+\left\|F^{\phi}\right\| \\
& =\int v^{*}(-\omega \oplus \omega)+\left\|H^{\phi}\right\| .
\end{aligned}
$$

Since the image of $v$ is contained in $\mathcal{U}$, the first term can be written as

$$
\int(\Phi \circ v)^{*}\left(d \Lambda_{\text {can }}\right)=\int_{\partial(\mathbb{R} \times[0,1])}(\Phi \circ v)^{*} \Lambda_{\text {can }} .
$$

By the boundary conditions this integral vanishes. Hence

$$
E_{\bar{J}}(v)=\left\|H^{\phi}\right\|=\operatorname{osc}\left(S_{\phi}\right) .
$$

We glue the trajectory $v=\left(v_{1}, v_{2}\right)$ into a curve to $M, u: \mathbb{R} \times S^{1} \rightarrow M$, as follows

$$
u(\tau, t)= \begin{cases}v_{1}(2 \tau, 2 t-1), & 0 \leq t \leq \frac{1}{2} \\ v_{2}(2 \tau, 1-2 t), & \frac{1}{2} \leq t \leq 1\end{cases}
$$


The map $u$ is smooth by elliptic regularity and satisfies

$$
\left\{\begin{array}{l}
\frac{\partial u}{\partial \tau}+J(u, t)\left(\frac{\partial u}{\partial t}-X_{H}(u)\right)=0 \\
u(\tau, t)=u(\tau, t+1) \\
\lim _{\tau \rightarrow \pm \infty} u(\tau, \cdot)=x^{ \pm}(\cdot) \\
u(0,0)=p
\end{array}\right.
$$

A straightforward chain rule calculation shows that $E_{J}(u)=E_{\bar{J}}(v)=\operatorname{osc}\left(S_{\phi}\right)$.

Now we come to the main step in the proof of Theorem B. Compare with [21, Proposition 9.6].

Proposition 4.2. There exists an element $u \in \mathcal{M}^{\text {local }}\left(\left[x^{-}, w^{-}\right],\left[x^{+}, w^{+}\right] ; p\right)$. Moreover, any local curve with these asymptotics satisfies $E_{J}(u)=\operatorname{osc}\left(S_{\phi}\right)$.

Proof. We remind the reader (see $[\mathbf{2}$, Lemma 7$]$ and $[\mathbf{1 3}$, Theorem 4.7]) that when $s$ is sufficiently small the thin part of the Floer homology satisfies

$$
H F_{2 n-j}^{\circ}\left(\Delta, s F ; \mathbb{Z}_{2}\right) \cong H_{j}\left(\Delta ; \mathbb{Z}_{2}\right) .
$$

Furthermore, the thin part of the Floer cap action $[\Delta] \cap_{s}^{\circ}(\cdot)$ agrees with the standard cap action of $[\Delta]$ and this map is an isomorphism. Let $j=2 n$ and 0 and consider the following diagram

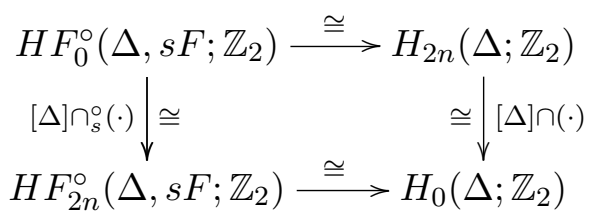

The thin cap action by the diagonal, the map $[\Delta] \cap_{s}^{\circ}(\cdot)$ in equation (4.1), shifts the grading by the dimension of $\Delta$.

Because $\Delta$ is orientable $H_{0}\left(\Delta ; \mathbb{Z}_{2}\right) \cong \mathbb{Z}_{2}$ and $H_{2 n}\left(\Delta ; \mathbb{Z}_{2}\right) \cong \mathbb{Z}_{2}$ and so the thin part of the cap product on the Floer homology $H F(\Delta, s F)$ is actually an isomorphism on $\mathbb{Z}_{2}$

$$
[\Delta] \cap_{s}^{\circ}(\cdot): \mathbb{Z}_{2} \stackrel{\cong}{\longrightarrow} \mathbb{Z}_{2}
$$

As such this map must be the identity. Hence it commutes with the projection $p^{+}: C F(\Delta, s F) \rightarrow C F^{+}(\Delta ; s F) \oplus C F^{\circ}(\Delta, s F)$ and Proposition 2.3 implies in combination with Theorem A that the composition $\Phi^{-} \circ[\Delta] \cap^{\mathcal{U}}(\cdot) \circ \Phi^{+}$is non-zero. Because all three maps are linear, this means that the local Floer cap product $[\Delta] \cap^{\mathcal{U}}(\cdot)$ is non-zero and therefore the moduli space $\mathcal{N}^{\mathcal{U}}\left(\left[\left(x^{-}, x^{-}\right), w^{-}\right],\left[\left(x^{+}, x^{+}\right), w^{+}\right] ; F^{\phi} ; \bar{J} ; p\right)$ must contain an odd number of elements, where $x^{ \pm}$are respectively the maximum and minimum points of $F$ and $w^{ \pm}$are any bounding disks. In particular, it 
is not empty. Let us denote this element $v$. By Lemma 4.1, there exists $u \in \mathcal{M}^{\text {local }}\left(\left[x^{-}, w^{-}\right],\left[x^{+}, w^{+}\right] ; q\right)$ and $E_{J}(u)=\operatorname{osc}\left(S_{\phi}\right)$.

Proof of Theorem B. We now know that any local curve $u \in \mathcal{M}^{\text {local }}\left(H^{\phi} ; p\right)$ satisfying

$$
\lim _{\tau \rightarrow \pm \infty} u(\tau, \cdot)=x^{ \pm}
$$

has energy equal to $\operatorname{osc}\left(S_{\phi}\right)$ and this collection is not empty.

On the other hand, if $u^{\prime} \in \mathcal{M}\left(\left[x^{-}, \widetilde{x^{-}}\right],\left[x^{+}, \widetilde{x^{+}}\right] ; H^{\phi} ; p\right)$ is any non-local curve, then we have

$$
E_{J}\left(u^{\prime}\right)=E_{J^{\prime}}\left(v^{\prime}\right),
$$

where $v^{\prime}(\tau, t)=\left(\phi_{H}^{t}\right)^{-1}\left(u^{\prime}(\tau, t)\right)$. One may check that the curve $v^{\prime}(\tau, t)=$ $\left(\phi_{H}^{t}\right)^{-1}\left(u^{\prime}(\tau, t)\right)$ satisfies the equation

$$
\frac{\partial v^{\prime}}{\partial \tau}+J^{\prime} \frac{\partial v^{\prime}}{\partial t}=0
$$

where $J^{\prime}=\left(\phi^{t}\right)^{*} J$. Since the periodic orbits of $X_{H^{\phi}}$ are all constant, the glued curve $v^{\prime} \# v$, where $v=v(\tau, t)=\left(\phi_{H}^{t}\right)^{-1}(u(\tau, t))$ and $u$ is local curve we constructed in Proposition 4.2 produces a non-constant pseudoholomorphic disk with boundary on the diagonal $\Delta$.

Therefore,

$$
\sigma(M, \omega)<E\left(v^{\prime} \# v\right)=E\left(v^{\prime}\right)+\left\|H^{\phi}\right\|
$$

Since we assume that

$$
\operatorname{osc}\left(S_{\phi}\right)=\left\|H^{\phi}\right\|<\frac{\sigma(M, \omega)}{2},
$$

it must be that $E_{J^{\prime}}\left(v^{\prime}\right)>\sigma / 2$. In short, $E_{J}\left(u^{\prime}\right) \geq\left\|H^{\phi}\right\|$.

Therefore, $A\left(\phi, J_{0} ; J ;\left[x^{-}, \widetilde{x^{-}}\right],\left[x^{+}, \widetilde{x^{+}}\right] ; p\right)=\left\|H^{\phi}\right\|=\operatorname{osc}\left(S_{\phi}\right)$. Since $H^{\phi}$ is quasi-autonomous, we then find $A\left(\phi, J_{0} ; J ; 1 ; p\right)=\operatorname{osc}\left(S_{\phi}\right)$. Therefore, the supremum over $p, J$ and $J_{0}$ is greater than the oscillation; in other words we have proven

$$
A(\phi ; 1) \geq \operatorname{osc}\left(S_{\phi}\right)
$$

This proves Theorem B.

Before proving Corollary $\mathrm{C}$, we prove a simple estimate between the $C^{1}$ norm of a Hamiltonian flow to the Hofer length of a generating Hamiltonian function. Let $J$ be any $\omega$-compatible almost complex structure and $g_{J}$ denote the corresponding metric. With respect to the metric $g_{J}$, the $C^{1}$ norm of the isotopy $\phi_{H}$ is

$$
\begin{aligned}
\left\|\phi_{H}\right\|_{C^{1}(M \times[0,1])} & :=\sup _{x, t}\left\|\left(T \phi_{H}^{t}\right)_{\mid x}\right\|+\sup _{x, t}\left\|\phi_{H}^{\dot{t}}(x)\right\| \\
& =\sup _{x, t}\left\|\left(T \phi_{H}^{t}\right)_{\mid x}\right\|+\sup _{x, t}\left\|X_{H}(x, t)\right\| .
\end{aligned}
$$


Lemma 4.3. For any Hamiltonian isotopy $t \mapsto \phi_{H}^{t}$

$$
\|H\| \leq \operatorname{diam}_{g_{J}}(M) \cdot\left\|\phi_{H}\right\|_{C^{1}(M \times[0,1])} .
$$

Proof. Let $d$ denote the Riemannian distance on $M$ and $x_{t}^{+}$and $x_{t}^{-}$be the points in $M$ where extreme values of $H(\cdot, t)$ occur.

$$
\max _{x \in M} H(x, t)=H\left(x_{t}^{+}, t\right) \text { and } \min _{x \in M} H(x, t)=H\left(x_{t}^{-}, t\right) .
$$

For $\epsilon>0$ choose a path $\sigma_{t}=\sigma_{t}(s):[0,1] \rightarrow M$ with

$$
\sigma_{t}(0)=x_{t}^{-}, \quad \sigma(1)=x_{t}^{+} \quad \text { and } \quad \int_{0}^{1}\left|\dot{\sigma}_{t}\right|_{g_{J}} d s<\epsilon+d\left(x_{t}^{-}, x_{t}^{+}\right) .
$$

Next

$$
\begin{aligned}
H\left(x_{t}^{+}, t\right)-H\left(x_{t}^{-}, t\right) & =\int_{0}^{1} \frac{d}{d s} H(\sigma(s), t) d s \\
& =\int_{0}^{1} g_{J}(\nabla H(\sigma, t), \dot{\sigma}) d s .
\end{aligned}
$$

By the Cauchy-Schwarz inequality, (4.4) is smaller than

$$
\|\nabla H\|_{L^{\infty}} \cdot\|\dot{\sigma}\|_{L^{1}} \leq\|\nabla H\|_{L^{\infty}}(d(x, y)+\epsilon) .
$$

Since $\epsilon>0$ is arbitrary, it follows that

$$
\|H\|_{\text {Hofer }} \leq\|\nabla H\|_{L^{\infty}} \cdot d\left(x_{t}^{-}, x_{t}^{+}\right) \leq\|\nabla H\|_{L^{\infty}} \cdot \operatorname{diam}_{g_{J}}(M) .
$$

Finally, the compatible almost complex structure $J$ is a $g_{J}$ isometry, hence $\|\nabla H\|=\left\|X_{H}\right\|$. This proves the Lemma.

Proof of Corollary C. A priori we know that

$$
\gamma(\phi) \leq\|\phi\|_{\text {Hofer }} \leq\left\|H^{\phi}\right\| \text {. }
$$

Let $\epsilon>0$ be such that $\operatorname{osc}\left(S_{\phi}\right)+\epsilon<\sigma(M, \omega) / 2$ and $\psi^{t}$ be a non-degenerate Hamiltonian isotopy with

$$
\left\|\phi^{t} \circ\left(\psi^{t}\right)^{-1}\right\|_{C^{1}(M \times[0,1])}<\frac{\epsilon}{\operatorname{diam}_{g_{J}}(M)} .
$$

Theorem B implies $\gamma(\psi)=\|\psi\|_{\text {Hofer }}=\left\|H^{\psi}\right\|$ and Lemma 4.3 implies

$$
\left\|H^{\phi} \# \overline{H^{\psi}}\right\|<\epsilon \text {. }
$$

By the triangle inequality,

$$
\begin{aligned}
\left\|H^{\phi}\right\| & \leq\left\|H^{\phi} \# \overline{H^{\psi}}\right\|+\left\|H^{\psi}\right\| \\
& <\epsilon+\left\|H^{\psi}\right\| \\
& =\epsilon+\gamma(\psi) \\
& \leq \epsilon+\gamma\left(\psi \circ \phi^{-1}\right)+\gamma(\phi) .
\end{aligned}
$$


By the symmetry property of the spectral norm, we next see that the above equals

$$
\begin{aligned}
& =\epsilon+\gamma\left(\phi \circ \psi^{-1}\right)+\gamma(\phi) \\
& \leq \epsilon+\left\|H^{\phi} \# \overline{H^{\psi}}\right\|+\gamma(\phi) .
\end{aligned}
$$

Hence

$$
0 \leq\left\|H^{\phi}\right\|-\gamma(\phi)<2 \epsilon .
$$

Letting $\epsilon$ approach zero proves $\left\|H^{\phi}\right\|=\gamma(\phi)$. Applying equation (4.5) a second time proves

$$
\gamma(\phi)=\|\phi\|_{\text {Hofer }}=\left\|H^{\phi}\right\| \text {. }
$$

\section{Concluding remarks}

It can be rather difficult to compute the constant $\sigma(M, \omega)$. For the twosphere with total surface area equal to $4 \pi$ then in fact $\sigma\left(S^{2} \times S^{2},-\omega \oplus \omega\right) / 2=$ $2 \pi$. The Hamiltonian function which generates the half-turn rotation about a diameter has Hofer length $2 \pi$. In this sense, the constant $\sigma / 2$ seems to be precise. This is plausible as rotation by more than half the way around a diameter is not Hofer (nor spectral) length minimizing: one can generate the same diffeomorphism by rotation in the opposite direction. However, it is not yet known if this situation is engulfable. In some cases, the Darboux neighborhood of the diagonal can be quite large. For the sphere the author speculates the largest possible Darboux neighborhood to be the compliment of the anti-diagonal in $S^{2} \times S^{2}$.

\section{References}

[1] M. Bialy and L. Polterovich, Geodesics of Hofer's metric on the group of Hamiltonian diffeomorphisms, Duke Math. J. 76 (1994), 273-292.

[2] Y.V. Chekanov, Lagrangian intersections, symplectic energy, and areas of holomorphic curves, Duke Math. J. 95 (1) (1998), 213-226.

[3] A. Floer, Morse theory for Lagrangian intersections, J. Diff. Geom. 28 (3) (1988), $513-547$.

[4] K. Fukaya and Y.-G. Oh, Zero-loop open strings and in the cotangent bundle and Morse homotopy, Asian J. Math. 1 (1) (1997), 96-180.

[5] K. Fukaya, Y.-G. Oh, H. Ohta and K. Ono, Lagrangian intersection Floer theory anomoly and obstruction, to be published in Int. Press, 2006.

[6] M. Gromov, Pseudo-holomorphic curves in symplectic manifolds, Invent. Math. 81 (1985), 307-347.

[7] H. Hofer, On the topological properties of symplectic maps, Proc. Royal Soc. Edinburgh Sect. A 115 (1990), 25-38.

[8] H. Hofer and D.A. Salamon, Floer homology and Novikov rings, in 'The Floer Memorial Volume' (H. Hofer, C.H. Taubes, A. Weintsein, E. Zehnder, eds.), 130, Birkhauser, 1995, 483-524. 
[9] F. Lalonde and D. McDuff, The geometry of symplectic energy, Ann. Math. 141 (2) (1995), 349-371.

[10] - Hofer's $L^{\infty}$ geometry: energy and stability of Hamiltonian flows I,II, Invent. Math. 122 (1) (1995), 1-33, 35-69.

[11] F. Laudenbach, Engouffrement symplectique et intersections lagrangiennes, Comm. Math. Helv. 70 (4) (1995), 558-614.

[12] D. McDuff, Geometric variants of the Hofer norm, J. Symplectic Geom. 1 (2002), 197-252.

[13] Y.-G. Oh, Floer cohomology, spectral sequences, and the Maslov class of Lagrangian embeddings, Inter. Math. Res. Notices 1996 (7) 305-346.

[14] - Gromov-Floer theory and disjunction energy of compact Lagrangian embeddings, Math. Res. Lett. 4 (1997), 895-905.

[15] - Symplectic topology as the geometry of the action functional, II pants product and cohomological invariants, Comm. Anal. Geom. 7 (1) (1999), $1-55$.

[16] - Chain level Floer theory and Hofer's geometry of the Hamiltonian diffeomorphism group, Asian J. Math. 6 (2002), 579-624.

[17] - Floer mini-max theory, the Cerf diagram and spectral invariants, 2004, preprint, arXiv: math.SG/0406449 v4.

[18] - Spectral invariants and the geometry of Hamiltonian diffeomorphisms, 2004, preprint, arXiv: math.SG/0403083 v1.

[19] - Construction of spectral invariants of Hamiltonian paths on closed symplectic manifolds, The breadth of symplectic and Poisson geometry: Festschrift in Honor of Alan Weinstein (Boston), 232, Progr. Math., Birkhauser, 2005, 525-570.

[20] - Normalization of the Hamiltonian and the action spectrum, J. Korean Math. Soc. 42 (2005), 65-83.

[21] - Spectral invariants, analysis of the Floer moduli space, and geometry of the Hamiltonian diffeomorphism group, Duke Math. J. 130 (2005), 199-295.

[22] Y. Ostrover, A comparison of Hofer's metrics on Hamiltonian diffeomorphisms and Lagrangian submanifolds, Comm. Contemp. Math. 5 (5) (2003), 1-9.

[23] L. Polterovich, Symplectic displacement energy for Lagrangian submanifolds, Erg. Th. Dyn. Sys. 13 (2) (1993), 357-367.

[24] J. Robbin and D.A. Salamon, Asymptotic behaviour of holomorphic strips, Ann. de l'Institute Henri Poincaré 18 (2001), 573-612.

[25] D. Salamon and E. Zehnder, Morse theory for periodic solutions of Hamiltonian systems and the maslov index, Comm. Pure Appl. Math. 45 (1992), 1301-1360.

[26] M. Schwarz, On the action spectrum for closed symplectically aspherical manifolds, Pacific J. Math. 193 (2000), 1046-1095.

[27] M. Usher, Spectral numbers in Floer theories, Compositio Math., to appear, preprint, arXiv: 0709.1127.

[28] I. Ustilovsky, Conjugate points on geodesics of Hofer's metric, Diff. Geom. Appl. 6 (4) (1996), 327-342.

[29] C. Viterbo, Symplectic topology as the geometry of generating functions, Math. Ann. 292 (4) (1992), 685-710. 


\author{
Department of Mathematics \\ Penn State University \\ UNIVERSITY PARK, PA \\ USA \\ E-mail address: spaeth@math.psu.edu
}

Received 08/06/2007, accepted 05/07/2008

This work formed a portion of my Ph.D. thesis. I wish to thank my advisor Yong-Geun Oh for his guidance and support. Theorem B is motivated by a version of that result announced in the preprint [18]. I also wish to thank Joel Robbin and Augustin Banyaga for many encouraging discussions. A final thanks goes to the referee whose comments significantly improved the article. 
